\title{
Effect of orthodontic forces on levels of enzymes in gingival crevicular fluid (GCF): A systematic review
}

Priyanka Kapoor ${ }^{1}$, Nitika Monga², Om Prakash Kharbanda², Sunil Kapila³, Ragini Miglani², Rajeswari Moganty ${ }^{4}$

DOI: https://doi.org/10.1590/2177-6709.24.2.40.e1-22.onl

Objective: Orthodontic force application releases multiple enzymes in gingival crevicular fluid (GCF) for activation, resorption, reversal, deposition of osseous elements and extracellular matrix degradation. The current systematic review critically evaluated all existing evidence on enzymes in orthodontic tooth movement. Methods: Literature was searched with predetermined search strategy on electronic databases (PubMed, Scopus, Embase), along with hand search. Results: Initial search identified 652 studies, shortlisted to 52 studies based on PRISMA. Quality assessment further led to final inclusion of 48 studies (13 moderately and 35 highly sensitive studies). Primary outcomes are significant upregulation in GCF levels of enzymes-aspartate aminotransferase (AST), alkaline phosphatase (ALP), matrix metalloproteinases (MMPs), lactate dehydrogenase (LDH), $\beta$-glucuronidase ( $\beta G$ ), tartrate resistant acid phosphatase (TRAP), acid phosphatase (ACP) and down regulation in cathepsin $\mathrm{B}(\mathrm{Cb})$. Site specificity is shown by ALP, TRAP, AST, LDH, MMP9 with levels at compression site increasing earlier and in higher quantities compared with tension site. ALP levels are higher at tension site only in retention. A positive correlation of LDH, ALP and AST is also observed with increasing orthodontic force magnitude. Conclusions: A strong evidence of variation in enzymes (ALP, AST, ACP TRAP, $\mathrm{LDH}, \mathrm{MMPs}, \mathrm{Cb}$ ) in GCF is found in association with different magnitude, stages and sites of orthodontic force application.

Keywords: Tooth movement. Gingival crevicular fluid (GCF). Enzymes. Systematic review.

Objetivo: a aplicação da força ortodôntica libera múltiplas enzimas no fluído crevicular gengival (FCG), desencadeando a ativação, reabsorção, reversão, deposição de elementos ósseos e degradação da matriz extracelular. A presente revisão sistemática avaliou criticamente toda a evidência disponível sobre os níveis de enzimas durante a movimentação ortodôntica. Métodos: utilizando-se estratégias predeterminadas, foram realizadas buscas em bases de dados eletrônicas (PubMed, Scopus, Embase), sendo também feitas buscas manuais. Resultados: a busca inicial identificou 652 estudos e, com base nas diretrizes do PRISMA, foram selecionados 52 estudos. A avaliação qualitativa resultou na inclusão final de 48 estudos (13 estudos com moderada sensibilidade e 35 com alto nível de sensibilidade). Os desfechos primários foram o aumento significativo dos níveis no FCG das enzimas aspartato aminotransferase (AST), fosfatase alcalina (FA), metaloproteinases de matriz (MMPs), lactato desidrogenase (LDH), $\beta$-glucuronidase $(\beta G)$, fosfatase ácido-resistente ao tartarato (TRAP), fosfatase ácida (FAC) e baixa regulação de catepsina B (Cb). Especificidade quanto ao local foi mostrada para FA, TRAP, AST, LDH e MMP9 com os níveis no lado de compressão aumentando mais rápido e em maiores quantidades, quando comparado ao lado de tensão. Os níveis de FA foram maiores no lado de tensão somente no período de contenção. Uma correlação positiva de LDH, FA e AST também foi observada à medida que a magnitude de força ortodôntica aumentou. Conclusões: há fortes evidências indicando que as variações nas enzimas (FA, AST, FAC, TRAP, LDH, MMPs, $\mathrm{Cb}$ ) presentes no FCG estão associadas a diferentes magnitudes, estágios e locais de aplicação da força ortodôntica.

Palavras-chave: Movimento dentário. Fluído crevicular gengival (FCG). Enzimas. Revisão sistemática.

${ }^{1}$ Jamia Millia Islamia, Faculty of Dentistry, Department of Orthodontics (New Delhi, India).

${ }^{2}$ All India Institute of Medical Sciences, Centre for Dental Education and Research, Division of Orthodontics and Dentofacial Deformities (New Delhi, India).

${ }^{3}$ University of California San Francisco, Division of Orthodontics

(San Francisco/CA, USA).

${ }^{4}$ All India Institute of Medical Sciences,Department of Biochemistry (New Delhi, India).

Submitted: July 17, 2018 - Revised and accepted: November 03, 2018
How to cite: Kapoor P, Monga N, Kharbanda OP, Kapila S, Miglani R, Moganty R. Effect of orthodontic forces on levels of enzymes in gingival crevicular fluid (GCF): A systematic review. Dental Press J Orthod. 2019 Mar-Apr; 24(2):40.e1-22. DOI: https://doi.org/10.1590/2177-6709.24.2.40.e1-22.onl

Contact address: Priyanka Kapoor

E-mail: pkapoor@jmi.ac.in - drkapoorpriya@gmail.com

» The authors report no commercial, proprietary or financial interest in the products or companies described in this article. 


\section{INTRODUCTION}

Orthodontic forces cause an initial inflammatory response followed by alterations in the vascular and neural envelope and perpetual bone and tissue remodelling accompanied by paracrine release of bioactive mediators. ${ }^{1-3}$ During orthodontic tooth movement (OTM), host-derived enzymes are released at various stages of activation, resorption, reversal and deposition of osseous elements and degradation of the extracellular matrix. ${ }^{4}$ Some of these enzymes have been identified in the periodontal (pdl) tissue of orthodontically moved teeth. ${ }^{5}$ Gingival crevicular fluid (GCF) is however a better choice for assessing biomolecules or mediators as sample collection is simple, sensitive, convenient, repetitive and noninvasive. ${ }^{6}$ Thus, the quantitative estimations of mediators in GCF reflect biochemical mechanisms associated with OTM. A systematic review (SR) by Kapoor et a ${ }^{6}$ in 2014 studied variation in GCF level of cytokines with type and magnitude of orthodontic forces and growth status of patients. It established a positive correlation of GCF activity index IL1RA (interleukin receptor antagonist)/ IL-1 3 ) with intensity of pain and velocity of OTM and a negative correlation with growth status of patients. Besides cytokines, numerous other mediators also alter GCF during OTM, comprehensively reviewed in SR by Alhadlaq ${ }^{3}$ in 2015. This SR highlighted working mechanisms of multiple mediators but heterogeneity of studies precluded attainment of concrete conclusions. Hence, the present SR aims to assess only a single family of mediators, enzymes, to establish their clinical correlations on sequential release in different phases of OTM and varying magnitude of orthodontic forces.

Soluble enzymes like lactate dehydrogenase (LDH) and aspartate aminotransferase (AST) present in cytoplasm are known to release in GCF only after cellular necrosis or hyalinization with heavy orthodontic forces. ${ }^{4}$ Tartrate-resistant acid phosphatase (TRAP) and alkaline phosphatase (ALP) exhibit osteoclastic and osteoblastic activity, respectively, ${ }^{1}$ and are identified in areas of tension (TS) or compression (CS) of teeth undergoing OTM. Heavy orthopedic forces of rapid maxillary expansion show an increase of $\beta$-glucuronidase (BG) lysosomal enzyme upon release from polymorphonuclear (PMN) leukocytes. ${ }^{7}$ Rise in PMN granules in surrounding tissues after fixed orthodontic appliance activation also show increase in myeloperoxidase (MPO) 2 hours (hr) after activation, traced both in GCF and saliva. ${ }^{8}$
The evidence on enzymes in OTM is plenty but scattered and lacks critical appraisal. Hence, the current SR is conducted to establish associations of enzymes in GCF to the site of application, magnitude and type of force, patient's growth status and the type of archwire ligation.

\section{MATERIAL AND METHODS \\ Protocol and registration}

The protocol for SR was registered in PROSPERO (www.crd.york.ac.uk/prospero, CRD42015017496) with a predetermined search strategy (Fig 1). It comprised of $\mathrm{MeSH}$ terms, Boolean terminology and free text terms with the keywords "enzyme" "protease", "orthodontic tooth movement" and "gingival crevicular fluid", together with several key enzymes. This search strategy was applied to key databases PubMed, Scopus and Embase in February 2018 with no language restrictions. Additional publications were identified through reference tracking and hand search of journals (Sains Malaysiana, Orthodontic Waves, Journal of Applied Sciences, APMC). The search was performed by two reviewers, followed by a cross-check by a third reviewer, in conformity with PRISMA, as shown in Figure 2.

\section{Evaluation of risk of bias/quality of individual studies}

The risk of bias, subjective to the included studies was measured by a customized Quality Assessment Instrument (QAI) ${ }^{6}$ based on QUADAS. This was objectively scored as minimally (scores of 1-12), moderately (13-20) and highly (21-29) sensitive, summarized in Table 1. No minimally sensitive studies were included in the review.

\section{RESULTS}

Were identified 102 articles in Pubmed, 460 in Scopus, 84 in Embase and 6 from hand search, in the initial search. Strict inclusion and exclusion criteria (Table 2) were applied after removing duplicates, resulting in 41 relevant articles. Five studies were further excluded: three studies whose full texts were not retrieved despite contacting the authors repeatedly through mail and academic social networking sites; one was a review on MMPs, and one had sample size smaller than inclusion criteria. Additional exclusion of three studies was done: two with QAI score smaller than 13, and one with a cross-sectional study design (Fig 2). 
((enzyme) OR (aspartate transaminase) OR (AST) OR (Acid phosphatase) OR (TRAP) OR (Alkaline Phosphatase) OR (ALP) OR (beta glucuronidase) OR ( $\beta$ glucuronidase) OR (matrix metalloproteinase) OR (MMP) OR (lactate dehydrogenase) or (LDH) OR (Cathepsin) or (myeloperoxidase) OR (MPO) OR (proteinase) OR (protease)) AND ((orthodontic force) OR (leveling) OR (orthodontic) OR (tooth movement) OR (maxillary expansion) OR (RME) OR (orthodontic tooth movement)) AND ((GCF) OR (gingival crevicular fluid))

\begin{tabular}{|c|c|}
\hline IDENTIFICATION & \\
\hline $\begin{array}{l}\text { Citations Screened from Electronic } \\
\text { Databases (646) } \\
\text { Pubmed-102 } \\
\text { Scopus- } 460 \\
\text { Embase-84 }\end{array}$ & $\begin{array}{l}\text { Records after Duplicates Removed } \\
\text { Pubmed-102 } \\
\text { Scopus-415 } \\
\text { Embase-31 } \\
\text { Handsearch- } 6\end{array}$ \\
\hline $\begin{array}{l}\text { Citation Screened from } \\
\underline{\text { Handsearching-6 }}\end{array}$ & \\
\hline 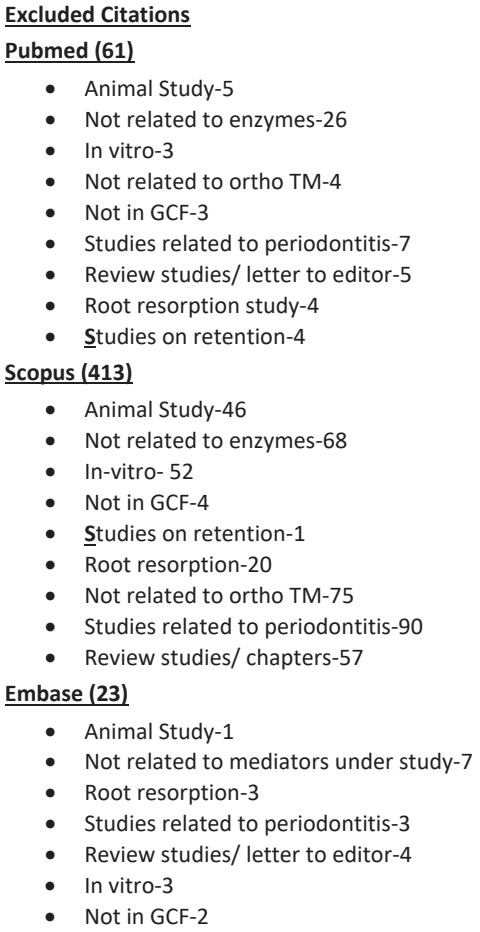 & $\begin{array}{l}\text { Exclusion based on non retrieval in full } \\
\text { text for detailed evaluation (5)- } \\
\text { Non retrieval of full text-3 (Chinese) } \\
\text { Review on MMPs-1 } \\
\text { Sample size less than inclusion criteria-1 }\end{array}$ \\
\hline
\end{tabular}

Figure 1 - Search strategy applied on databases for inclusion of studies in the review.
Figure 2 - PRISMA flow diagram for inclusion of studies in the systematic review
PRISMA finally resulted in 48 publications in total, with consensus among all reviewers. The QAI of these studies indicated 13 moderately sensitive and 35 highly sensitive studies.

Data extraction of shortlisted studies ${ }^{7-54}$ (for participant characteristics and study design are as follows (Table 3):

» Sample size: Sample size was categorized in three groups, $\leq 15(\mathrm{n}=22), 15-20 \quad(\mathrm{n}=15), \geq 21 \quad(\mathrm{n}=10)$ and one study each having sample of five subjects ${ }^{27}$ and 99 subjects. ${ }^{21}$

"Sex predilection: Forty- one studies mentioned sex distribution in the sample, two of which had female subjects only, ${ }^{24,36}$ and five had equal numbers of male and female subjects. ${ }^{10,19,23,29,43}$

" Age predilection: Studies used age as either range or mean with standard deviation in all studies; one study considered two separate age groups of adolescents and adults. ${ }^{15}$ 
Table 1 - Inclusion and Exclusion criteria applied for inclusion of studies in the systematic review.

\begin{tabular}{|c|c|c|c|}
\hline Criteria & Sub criteria & Inclusion & Exclusion \\
\hline \multirow{5}{*}{$\begin{array}{l}\text { Participants/ } \\
\text { population }\end{array}$} & Type of sample & Human studies & Animal studies, in vitro studies \\
\hline & Age groups & if specified & Not mentioned \\
\hline & Male to female ratio & if specified & Not mentioned \\
\hline & Controls & present (either internal /external) & No controls \\
\hline & Sample size (sample size, not number of teeth studied) & $\geq 5$ & $<5$ \\
\hline \multirow{7}{*}{$\begin{array}{l}\text { Intervention(s), } \\
\text { exposure(s) }\end{array}$} & Mediators studied & $\begin{array}{c}\text { enzymes [AST, MPO, ALP,BG, LDH, } \\
\text { CatB, Cs, CAMP RII, MMPs] }\end{array}$ & $\begin{array}{l}\text { Other than enzymes (cytokines/ } \\
\text { hormones/PGs) }\end{array}$ \\
\hline & Exposure & $\begin{array}{l}\text { Orthodontic force application in } \\
\text { healthy patients }\end{array}$ & $\begin{array}{l}\text { Studied in periodontal inflammation/ } \\
\text { root resorption/ not related }\end{array}$ \\
\hline & Orthodontic mechanics & Specified & Not specified \\
\hline & Oral hygiene regimen & Mentioned & Not mentioned \\
\hline & Use of antibiotic/anti-inflammatory drugs & Not used & Not mentioned/ used \\
\hline & Medium of study & GCF & $\begin{array}{l}\text { Other than GCF/ peri-implant fluid/ } \\
\text { saliva }\end{array}$ \\
\hline & GCF sample collection instrument & $\begin{array}{l}\text { Periopaper/micropipette/ endodontic } \\
\text { paper }\end{array}$ & Not mentioned \\
\hline
\end{tabular}

AST: aspartate transaminase, MPO: myeloperoxidase, ACP: acid phosphatase, ALP: alkaline phosphatase, $\beta$ G: $\beta$ glucuronidase, LDH: lactate dehydrogenase, CatB: cathepsin B, Cs: caspase, CAMP RIl:cyclic adenosine monophosphate (AMP)-dependent protein kinase subunit (RII), PGs: prostaglandins, MMPs: matrix metalloproteinases.

" Number of studies reporting enzymes: Alkaline phosphatase was evaluated in maximum number of studies $(n=17)$, closely followed by AST in 10, matrix metalloproteinases (MMPs) in eight, LDH in six, MPO in five and TRAP in four and acid phosphatase (ACP) in three studies. Two studies studied $\beta G$, cathepsin (Cp) and tissue inhibitor of MMPs (TIMPs) each. Single studies evaluated cystatin (Cys) and thrombospondin1 (TSP1). Additionally, granulocyte-macrophage colony-stimulating factor (GMCSF), epidermal growth factor (EGF), macrophage inflammatory protein-1 $\beta$ (MIP-1 $\beta$ ), methyl-accepting chemotaxis protein-1 (MCP-1), chemokine RANTES (Regulated on activation normal $\mathrm{T}$ cells expressed and secreted) were evaluated as secondary outcomes.

"Study duration: The duration of studies ranged from $8 \mathrm{hr}$ to 24 weeks (wk) to the maximum of one year (y). One study each was done for $8 \mathrm{hr}, 1 \mathrm{wk}$, 5 month $(\mathrm{m})$ and $1 \mathrm{y}$ duration, two studies for $6 \mathrm{~m}$, three for $2 \mathrm{~m}$, five each for $2 \mathrm{wk}$ and $3 \mathrm{~m}$, eight for $3 \mathrm{wk}, 15$ for approximately $1 \mathrm{~m}$. One study did not specify duration - only completion of alignment.

» Observation intervals for GCF collection: Studies had GCF collection at repeated observation time points (OTP) ranging from 2 times $^{28}$ to 31 times (each day of the month). ${ }^{27}$ Six OTPs were taken in 16 studies, closely followed by 4 OTPs in 15 studies, 9 OTPs in nine studies, 3 and 10 OTPs in two studies each, 2, 7, 8 and 31 OTPs in single study each.
"Site for GCF collection: Forty one studies specified mesial or distal or buccal site for GCF collection while seven studies mentioned the tooth but not the site for sample retrieval. The technique by Lamster et al..$^{55}$ utilizing six sites was used in four studies. $10,19,33,44,47$

" Mechanics of force: Studies used continuous force both for tooth retraction (26 studies) and leveling of arches (13 studies). Retraction involved 19 studies using NiTi coil spring, two using steel ligature lacebacks, three using NiTi push coil spring, and one study each for $\mathrm{V}$ loop and $\mathrm{NiTi}$ open coil spring. Besides, nine studies used intermittent orthodontic/orthopaedic forces, employing elastomeric chain for retraction in five, Hyrax for expansion in three, and TMA spring for intrusion in one study.

" The level of force: Only 33 studies mentioned force levels for OTM. The level of forces ranged from $50 \mathrm{~g}, 50-75 \mathrm{~g}, 100-150 \mathrm{~g}, 16 \mathrm{~N} / \mathrm{turn}, 1-1.5 \mathrm{~N}, 200 \mathrm{cN}$, $400 \mathrm{~g}$ in one study each, $125 \mathrm{~g}$ in three, $100 \mathrm{~g}$ in six, $250 \mathrm{~g}$ in eight and $150 \mathrm{~g}$ in seven studies. Few studies had different treatment groups employing variable magnitudes of force. . $^{91,34,35,36}$

\section{Oral hygiene regimen and gingival health assessment (Table 4)}

Professional oral prophylaxis was done before treatment in 34 studies and at every OTP in 16 studies, but was not mentioned in 12 studies. Verbal edification for oral hygiene maintenance was done in 33 studies. 
Table 2 - Quality Assessment Instrument (QAI) customized from QUADAS (Quality Assessment of Diagnostic Accuracy Studies) tool for assessment of risk of bias for inclusion of studies in the review.

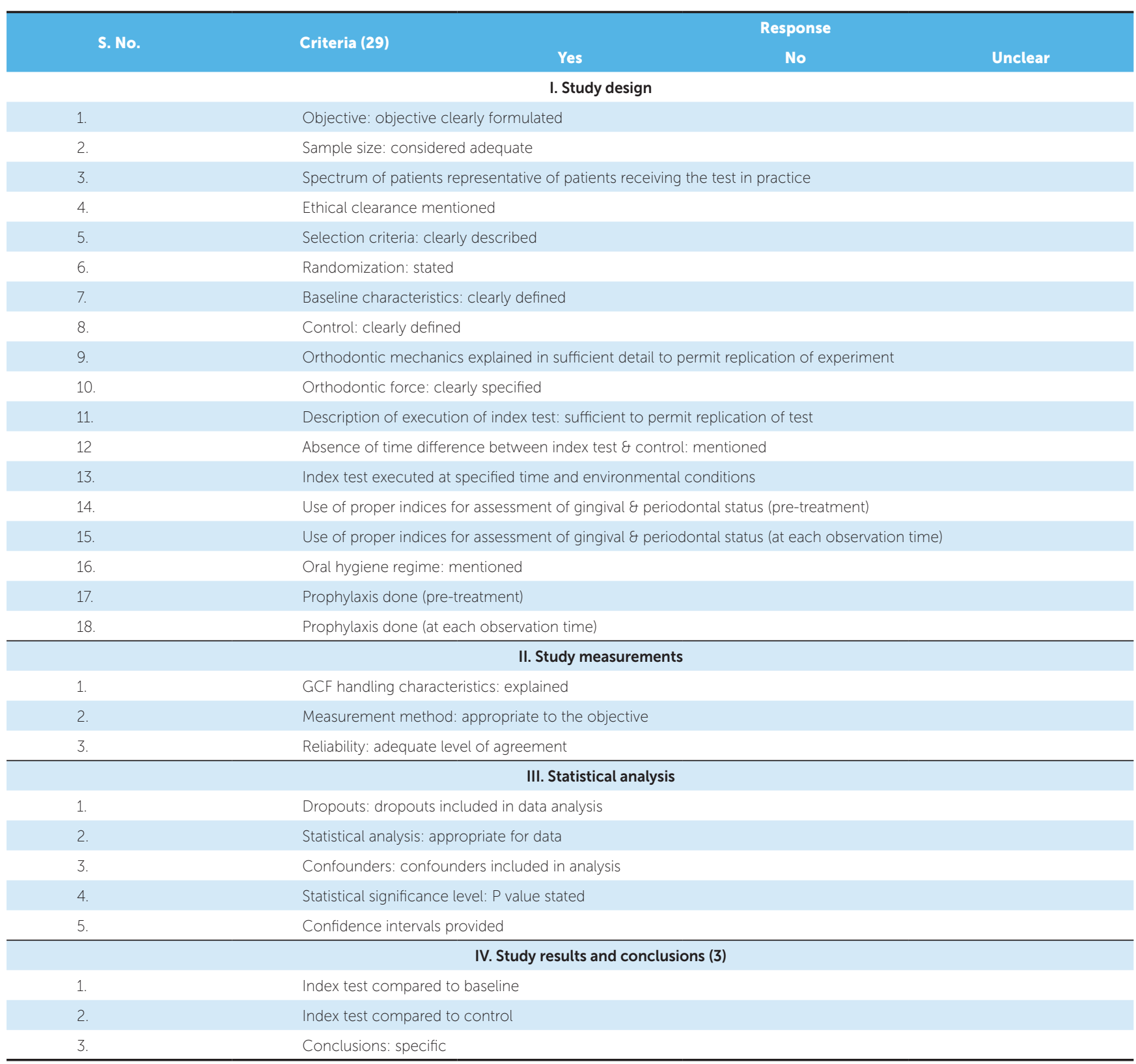

*Index test: Refers to collection of GCF at each observation interval in treatment teeth.

Nine studies advocated chlorhexidine mouthwash and two studies, benzydamine hydrochloride; but six studies refrained the use of any mouthwash during study period. Gingival and pdl health evaluation was done before treatment in 31 studies and at every OTP in 24 studies using "Quigley Hein Index" for visual plaque or its Turesky modification, Eastman interdental bleeding score, generalized probing depths $<3 \mathrm{~mm}$, radiographic evidence of pdl bone loss, gingival recession, full-mouth plaque score or full-mouth bleeding score (<20\%).

\section{GCF characteristics (Table 5)}

" GCF collection: GCF was collected by Periopaper (OraFlow, Plainview, New York, NY, USA) in 32 studies, micropipette in seven, filter paper in two, paper point in two and endodontic paper strip in five studies. Time of sample collection, room temperature and humidity conditions were specified in three studies each.

" GCF handling: Depth of Periopaper insertion was $1 \mathrm{~mm}$ in 21 studies, $1-2 \mathrm{~mm}$ in two, and $2 \mathrm{~mm}$ in one study. Duration of GCF collection was 30 seconds (s) in 21 studies, 60s in 13 studies and 10s, 3 minutes (min) 
Table 3 - Participant and study characteristics table.

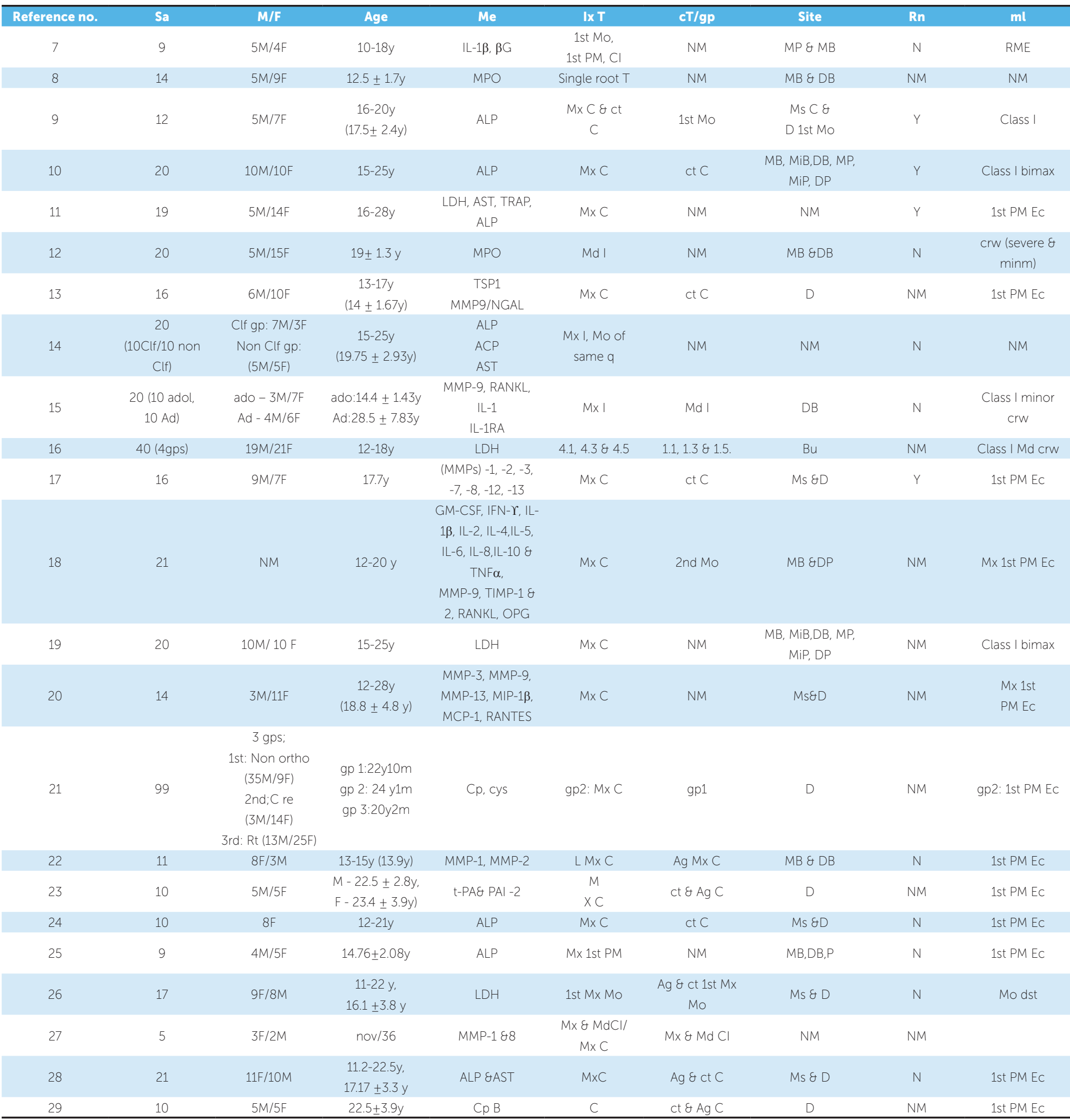

A-article, f-force, t/o-type of, mc-mechanics, md/mc-mode of mechanics, tm- time, a-appliance, re-reactivation, to-total, du-duration, $n$-number, ob-observation, B-baseline, min- minutes, g- grams, Ir- Interrupted, Cn- Continuous, Im- intermittent, Rt-retraction, sg-segmented, sp-spring, Ech-elastomeric chain, NiTi-nitinol, c-control, NM-not mentioned, y-year, d-day, m-month, h-hour, lv-levelling, se-separator, ac-activated, HG-headgear, NHG-non-headgear, bu-buccal, la-labial, RME-rapid maxillary expansion, HR-hybrid retractor, RCD- rapid canine distaliser, Sa-Sample, M/F-male/female, E- enzyme, Me- mediator, T-tooth, sc-specification, rn-randomisation, ml-malocclussion, HS-Handsearched, P-Pubmed, S-Scopus, GS- Google scholar, N-No, Y-yes, Mx-Maxilla, Md-Mandible, H-history, Is-loss, gvgingival, if-inflammation, PD-probing depth, wk-week, R-right, L-left, C-canine, PM-premolar, Mo-molar, Cl-central incisor, l-incisor, Ag- Antagonistic, ct- Contralateral, ip-interproximal, op-opposing, Ex- Experimental, c- Control, aj-adjacent, Exs-Experimental site1, Ec- Extraction, Ms- Mesial, D- Distal, rq-required, q-quadrant, OTM-orthodontic tooth movement, sf- surface, ado-adolescent, AST-aspartate transaminase, TRAP-Acid phosphatase, ALP-Alkaline Phosphatase, $\beta$ G- beta glucuronidase, MMP-matrix metalloproteinase, LDH-lactate dehydrogenase, Cp-Cathepsin, MPO- myeloperoxidase, CK-creatinine, NO-Nitric oxide, IL-Interleukin, 


\begin{tabular}{|c|c|c|c|c|c|c|c|c|c|}
\hline$F$ & $t / 0 f$ & $m e$ & $\mathrm{md} / \mathrm{mc}$ & re & to du & No. / ob & $\mathrm{tm} / \mathrm{ob}$ & B & $B=c$ \\
\hline NM & $\operatorname{Im} F$ & RME & Hyrax & Y & $81 \mathrm{~d}$ & 10 & $\begin{array}{c}0,14,25,32,33,39 \\
46,53,60,81 \mathrm{~d}\end{array}$ & $14 d$ & Y \\
\hline NM & $\mathrm{Cn}$ & Lv & Arch wi & $\mathrm{N}$ & $14 d$ & 4 & $-7 d, 0,2 h, 7,14 d$ & 0 & Y \\
\hline $\begin{array}{l}\text { 1st q: } 150 \mathrm{cN} \text {, } \\
\text { 2nd q: } 50 \mathrm{cN} \text {, } \\
100 \mathrm{cN}, 150 \mathrm{cN}\end{array}$ & $\mathrm{Cn}$ & Rt & NiTisp & NA & $3 w k$ & 4 & $0,1 w k, 2 w k, 3 w k$ & 0 & N \\
\hline $125 \mathrm{~g}$ & $\mathrm{Cn}$ & Rt & NiTi sp & NA & $3 w k$ & 6 & $0,1 \mathrm{~h}, 24 \mathrm{~h}, 7 \mathrm{~d}, 14 \mathrm{~d}, 21 \mathrm{~d}$ & 0 & N \\
\hline $100 / 150 \mathrm{~g}$ & $\mathrm{Cn}$ & Rt & NiTisp & NA & $5 w k$ & 6 & $0,1,2,3,4,5 \mathrm{wk}$ & 0 & Y \\
\hline NM & $\mathrm{Cn}$ & LV & Arch wi lig & NA & $2 w k$ & 4 & $0,2 h, 7 d, 14 d$ & 0 & Y \\
\hline NM & $\mathrm{Cn}$ & Rt & LB & NA & $2 w k$ & 8 & $\begin{array}{c}-1 h,+1 h, 4,8,24,72 h, 1 w k \\
2 w k\end{array}$ & $-1 h$ & N \\
\hline $150 \mathrm{cN}$ & $\mathrm{Cn}$ & LV & NiTi wi & NA & $30 d$ & 5 & $0,3,9,15,30 d$ & 0 & Y \\
\hline NM & $\mathrm{Cn}$ & LV & NiTi wi & Y & $20 w k$ & 4 & $0,3,6,18,20 w k$ & 0 & Y \\
\hline NM & $\mathrm{Cn}$ & LV & NiTi \& thrm wi & Y & $42 d$ & 6 & $-2 w k, 0,1 h, 7,28,42 d$ & $-2 w k$ & N \\
\hline $150 \mathrm{~g}$ & $\mathrm{Cn}$ & Rt & NiTisp & N & $21 d$ & 6 & $0,1,24 h, 7,14,21 d$ & 0 & N \\
\hline $100 \mathrm{~g}$ & $\mathrm{Cn}$ & Rt & NiTisp & N & $42 d$ & 4 & $-10 w k, 0,4 h, 7 d, 42 d$ & 0 & N \\
\hline $125 \mathrm{~g}$ & $\mathrm{Cn}$ & Rt & NiTi sp & $\mathrm{N}$ & $21 d$ & 5 & $0,1 \mathrm{~h}, 7,14,21 \mathrm{~d}$ & 0 & Y \\
\hline $150 \mathrm{~g}$ & $\mathrm{Cn}$ & Rt & V-loop \& NiTi sp & $\mathrm{N}$ & $87 d$ & 7 & $-7 d, 0,1 h, 24 h, 14,21,80 d$ & 0 & Y \\
\hline
\end{tabular}

\begin{tabular}{|c|c|c|c|c|c|c|c|c|c|}
\hline $100-150 \mathrm{~g}$ & Imf & Rt & $\mathrm{ECh}$ & NM & $1 \mathrm{~m}$ & 4 & $0,1 \mathrm{~d}, 1 \mathrm{wk}, 1 \mathrm{~m}$ & 0 & $\mathrm{~N}$ \\
\hline $150 \mathrm{~g}$ & $\mathrm{Cn}$ & Rt & NiTi sp & $\mathrm{N}$ & $8 \mathrm{~h}$ & 5 & $0,1 h, 2 h, 3 h, 4 h, 8 h$ & 0 & $\mathrm{~N}$ \\
\hline $250 \mathrm{~g}$ & Imf & Rt & Ech & NM & $168 \mathrm{~h}$ & 4 & $0,1,24,168 \mathrm{~h}$ & 0 & $\mathrm{~N}$ \\
\hline $100 \mathrm{~g}$ & $\mathrm{Cn}$ & Rt & NiTi sp & $\mathrm{N}$ & $21 d$ & 6 & $-1,0,1,7,14,21 d$ & -1 & $\mathrm{~N}$ \\
\hline $50 \mathrm{~g}$ & Imf & Intr & TMA sp & Y & $28 d$ & 5 & $\begin{array}{c}0,1,24,168 h, 22 d, 28 d \\
\text { reac- } 21 d\end{array}$ & 0 & Y \\
\hline $250 \mathrm{~g}$ & $\mathrm{Cn}$ & Rt & NiTi sp & N & $21 d$ & 4 & $0,7,14$ \& 21d & 0 & $\mathrm{~N}$ \\
\hline NM & $\mathrm{Cn}$ & aln & NM & N & $30 d$ & 31 & 0,1-30d,once/d for $1 \mathrm{~m}$ & 0 & $\mathrm{~N}$ \\
\hline $150 \mathrm{~g}$ & $\mathrm{Cn}$ & Rt & NiTi sp & $\mathrm{N}$ & $28 d$ & 2 & $0,28 d$ & 0 & $\mathrm{~N}$ \\
\hline $250 \mathrm{~g}$ & Imf & Rt & Ech & NM & $168 \mathrm{~h}$ & 4 & $0,1 \mathrm{~h}, 24 \mathrm{~h}, 168 \mathrm{~h}$ & 0 & $\mathrm{~N}$ \\
\hline
\end{tabular}

CRP- C Reactive Protein, hm-humidity, sc-specification, ins-insertion, MB-Mesio-buccal, ML-Mesio-lingual, DP-Disto-palatal, DB- Disto-buccal, df-differentiation, gp-group, cmp-compression, kPa-kilopascal, mx-maximum, gw-growth, Oc-osteoclast, Ix- Index, Bu Tp-buccal tipping, C-canine, Clf-cleft, I-incisor, NA-not applicable, wk-week, crw-crowding, minm-minimum, bimax-bimaxillary, wi-wire, lig-ligature, Ad-adult, RANKL-receptor antagonist nuclear kappa ligand, OPG-osteoprotegerin, IL-_1RA-interleukin 1 receptor antagonist, therm-thermoplastic, t-PA-plasminogen, TNF $\alpha$-tumour necrosis factor, TIMP-Tissue inhibitor metalloproteinase, MCP- Methyl-accepting chemotaxis protein, MPO-myeloperoxidase, ortho-orthodontic, cys-cysteine, cN-centinewton, TSP-thrombospondin 1, NGAL-neutrophil gelatinase-associated lipocalin, ACP-acyl carrier protein, CS-chondroitin sulphate, GM-CSF- Granulocyte-macrophage colony-stimulating factor, IFN $\gamma$-Interferon gamma, MIP-Macrophage inflammatory protein, $\beta$ G-beta globulin, PAI-plasminogen activator inhibitor, EGF-Epidermal growth factor, dst-distalisation, Intr-intrusion, aln-alignment, cst-constriction, AL-after loading, BL-before loading, Mx constr- Maxillary constriction, Exp- Expansion, Hyr- Hyrax, LB-laceback, TB-Tie back, SE-superelastic NiTi, HANT- heat-activated NiTi, MSSS- multistranded stainless steel. 
Table 3 - continuation - Participant and study characteristics table.

\begin{tabular}{|c|c|c|c|c|c|c|c|c|c|}
\hline Ref No. & $\mathrm{Sa}$ & M/F & Age & Me & Ix T & cT/gp & Site & Rn & ml \\
\hline 30 & 16 & $10 \mathrm{~F} / 6 \mathrm{M}$ & $\begin{array}{c}11-21 y \\
15.5 \pm 3.5 y\end{array}$ & ALP & Mx 1st Mo & ct $\& A g$ 1st Mo & Ms \& D & NM & Mo dst \\
\hline 31 & 9 & $5 \mathrm{M} / 4 \mathrm{~F}$ & 10-18y & $\mathrm{IL}-1 \beta, \beta \mathrm{G}$ & $\begin{array}{l}\text { Mx 1st Mo, } \\
1 \text { st PM \& Cl }\end{array}$ & NM & $M B \& M P$ & NM & Mx cst \\
\hline 32 & 12 & $3 \mathrm{M} / 9 \mathrm{~F}$ & $14.4 \pm 0.9 y$ & $\begin{array}{c}\text { IL-1 } \beta, \text { IL-6, TNF- } \alpha \\
E G F, \beta 2-\mu G\end{array}$ & C & $\mathrm{Ag} \mathrm{C/Ct} \mathrm{C}$ & D & NM & 1st PM Ec \\
\hline 33 & 9 & NM & $13-17 y$ & $\begin{array}{l}\text { TRAP5b, } \\
\text { IL-10,TNF- } \alpha\end{array}$ & $M x \& M d C$ & ct $C$ & $\begin{array}{l}M B, M i B, M P \& \\
D B, M i P, D P\end{array}$ & NM & 1st PM Ec \\
\hline 34 & 19 & $13 F / 6 M$ & $16-28 y$ & ALP, AST, TRAP & $R \& L M x C$ & NM & D & NM & Mx 1st PM Ec \\
\hline 36 & 12 & $11 \mathrm{~F} / 1 \mathrm{M}$ & $14-24 y$ & TRAP & $M \times C$ & BS & Ms \&D & Y & 1st PM Ec \\
\hline 37 & 14 & $4 \mathrm{M} / 10 \mathrm{~F}$ & $15-27 y$ & ALP & $M \times C$ & NM & Ms \&D & NM & crw $(4-8 \mathrm{~mm})$ \\
\hline 38 & 10 & $8 \mathrm{~F} / 2 \mathrm{M}$ & $15-27 y$ & ALP & $M \times C$ & NM & Ms \&D & NM & 1st PM Ec \\
\hline 39 & 13 & NM & $\begin{array}{c}14.4 \pm 3.7 y \\
23.3 \pm 4 y\end{array}$ & AST & $M \times C$ & ct $C$ & Ms \&D & NM & 1st PM Ec \\
\hline 40 & $\begin{array}{c}13 \\
\text { (6ado, 7Ad) }\end{array}$ & NM & $\begin{array}{c}14.4 \pm 3.7 y \\
23.3 \pm 4 y\end{array}$ & AST & $M \times P M$ & Ag PM & Ms $\& D$ & NM & 1st PM Ec \\
\hline 41 & 22 & $12 F / 10 M$ & $13-22 y$ & AST & $M \times C$ & NM & Ms \& D & Y & NM \\
\hline 42 & 12 & $7 F / 5 M$ & $14 \pm 2 y$ & $A C P, A L P$ & $M \times C$ & $\mathrm{Ag} \mathrm{C}, \mathrm{ct} C$ & Ms \& D & Y & 1st PM Ec \\
\hline 43 & 10 & $5 F / 5 M$ & $15-20 y$ & ALP & $\begin{array}{c}M \times C, M \times 2 n d \\
P M\end{array}$ & NM & $\begin{array}{l}D \text { of } C \& M s \text { of } \\
\text { 2nd PM }\end{array}$ & NM & 1st PM Ec \\
\hline 44 & 23 & $15 \mathrm{~F} / 8 \mathrm{M}$ & $9 \pm 1.4 y$ & ALP & Mx rt \& lt 1st M & $\mathrm{Ag}$ ist $\mathrm{M}$ & $\begin{array}{c}M B, M i B, D B, M P \\
M i P, D P\end{array}$ & NM & Mx constr \\
\hline 45 & 10 & $7 F / 3 M$ & $14-27 y$ & ALP & Rt $M \times C$ & Lt Mx C & MS, D & NM & 1st PM Ec \\
\hline 46 & 7 & $5 F / 2 M$ & $14-27 y$ & $\mathrm{ACP}$ & Rt Mx C & Lt Mx C & MS, D & NM & 1st PM Ec \\
\hline 47 & 20 & $9 \mathrm{~F} / 11 \mathrm{M}$ & $12-25 y$ & LDH & $M \times C$ & ct $C$ & $\begin{array}{c}M B, M i B, D B, M P \\
M i P, D P\end{array}$ & NM & 1st PM Ec \\
\hline 48 & 18 & $10 \mathrm{~F} / 8 \mathrm{M}$ & nov/22 & AST & Max 1st Mo & ct \& Ag 1st Mo & Ms \& D & $N$ & Mo dst \\
\hline 49 & 20 & $6 F / 4 M$ & $20.6 \pm 3.2 y$ & ALP & $M \times C, M d C$ & BS & D & Y & 1st PM Ec \\
\hline 50 & 55 & $28 \mathrm{~F} / 27 \mathrm{M}$ & $15.1(1.7)$ & $\begin{array}{c}\text { Adiponectin, } \\
\text { Leptin, Resistin, } \\
\text { MPO, CRP, } \\
\text { MMP 8,9, } \\
\text { TIMP1, MMP8/ } \\
\text { TIMP1, MMP9/ } \\
\text { TIMP1,RANKL }\end{array}$ & $\begin{array}{c}\text { Mand } 6 \\
\text { anterior teeth }\end{array}$ & $\begin{array}{l}\text { Normal weight } \\
\text { children }\end{array}$ & D & NM & Non Ec \\
\hline 51 & 22 & $14 F / 8 M$ & $11-21 y$ & ALP & $\begin{array}{l}\text { Max 1st - } \\
\text { Mrt \&Lt }\end{array}$ & $\begin{array}{l}\text { Mand 1st - } \\
\text { Mrt \& Lt }\end{array}$ & NM & $M B$ & Exp \\
\hline 52 & 60 & $41 F / 19 M$ & $18 \pm 1.5$ & MPO & Mand $\mathrm{Cl}$ & BS & NM & NM & $\begin{array}{c}4-6 \mathrm{~mm} \text { mand I } \\
\text { crow }\end{array}$ \\
\hline 53 & 45 & NM & $6.25,5.6,6.10$ & MPO & Mand I & BS & NM & Y & $\begin{array}{c}4-6 \mathrm{~mm} \text { mand I } \\
\text { crow }\end{array}$ \\
\hline 54 & 30 & NM & $9-15 y$ & AST & Rt $M \times P M$ & Lt Mx PM & NM & NM & NM \\
\hline
\end{tabular}

A-article, f-force, t/o-type of, mc-mechanics, md/mc-mode of mechanics, tm- time, a-appliance, re-reactivation, to-total, du-duration, n-number, ob-observation, B-baseline, min- minutes, g- grams, Ir- Interrupted, Cn- Continuous, Im- intermittent, Rt-retraction, sg-segmented, sp-spring, Ech-elastomeric chain, NiTi-nitinol, c-control, NM-not mentioned, y-year, d-day, m-month, h-hour, lv-levelling, se-separator, ac-activated, HG-headgear, NHG-non-headgear, bu-buccal, la-labial, RME-rapid maxillary expansion, HR-hybrid retractor, RCD- rapid canine distaliser, Sa-Sample, M/F-male/female, E- enzyme, Me- mediator, T-tooth, sc-specification, rn-randomisation, ml-malocclussion, HS-Handsearched, P-Pubmed, S-Scopus, GS- Google scholar, N-No, Y-yes, Mx-Maxilla, Md-Mandible, H-history, Is-loss, gvgingival, if-inflammation, PD-probing depth, wk-week, R-right, L-left, C-canine, PM-premolar, Mo-molar, Cl-central incisor, I-incisor, Ag- Antagonistic, ct- Contralateral, ip-interproximal, op-opposing, Ex- Experimental, c- Control, aj-adjacent, Exs-Experimental site1, Ec- Extraction, Ms- Mesial, D- Distal, rq-required, q-quadrant, OTM-orthodontic tooth movement, sf- surface, ado-adolescent, AST-aspartate transaminase, TRAP-Acid phosphatase, ALP-Alkaline Phosphatase, $\beta$ G- beta glucuronidase, MMP-matrix metalloproteinase, LDH-lactate dehydrogenase, Cp-Cathepsin, MPO- myeloperoxidase, CK-creatinine, NO-Nitric oxide, IL-Interleukin, 


\begin{tabular}{|c|c|c|c|c|c|c|c|c|c|}
\hline $\mathbf{F}$ & $t / o f$ & mc & $\mathrm{md} / \mathrm{mc}$ & re & to du & No. / ob & $\mathrm{tm} / \mathrm{ob}$ & B & $B=c$ \\
\hline $250 \mathrm{~g}$ & $\mathrm{Cn}$ & dst & NiTi sp & NM & 4 wk & 6 & $0,1 \mathrm{~h}, 1,2,3,4 \mathrm{wk}$ & 0 & $\mathrm{~N}$ \\
\hline NM & ImF & RME & Hyrax & Y & $74 d$ & 10 & $\begin{array}{c}\mathrm{BL}-0(\mathrm{~B}, \mathrm{O} 1), 14 \mathrm{~d}(\mathrm{c}, \mathrm{O} 2) \\
\mathrm{Al-4d}(\mathrm{O}), 11 \mathrm{~d}(\mathrm{O}), 12 \mathrm{~d}(\mathrm{O}), \\
\text { 18d (O6), 19d (O7), 25d (O8), } \\
\text { 32d (O9), 39d (10), 60d (O11) }\end{array}$ & 0 & $\mathrm{~N}$ \\
\hline $250 \mathrm{~g}$ & $\operatorname{Imf}$ & Rt & Ech & N & $7 d$ & 4 & $0,1,24,168 \mathrm{~h}$ & 0 & $\mathrm{~N}$ \\
\hline $150 \mathrm{~g}$ & $\mathrm{Cn}$ & Rt & NiTi sp & NM & $28 d$ & 5 & $0,1 h, 24 h, 7 d, 28 d$ & 0 & $N$ \\
\hline $\begin{array}{l}\text { gp1-100g } \\
\text { gp 2-150g }\end{array}$ & $\mathrm{Cn}$ & Rt & NiTi sp & NM & $5 \mathrm{wk}$ & 6 & $0,1 w k, 2 w k, 3 w k, 4 w k, 5 w k$ & 0 & Y \\
\hline $1 \mathrm{~N} \& 1.5 \mathrm{~N}$ & $\mathrm{Cn}$ & Rt & NiTi sp & NM & $5 w k$ & 6 & Owk, 1wk, 2wk, 3wk, 4wk, 5wk & 0 & Y \\
\hline $100 / 150 \mathrm{~g}$ & $\mathrm{Cn}$ & Rt & NiTi pushsp & $N$ & $5 w k$ & 6 & $0,1,2,3,4,5 \mathrm{wk}$ & 0 & Y \\
\hline NM & $\mathrm{Cn}$ & $\begin{array}{l}\text { lv, } \\
\text { aln }\end{array}$ & NiTi wi & $N$ & $3 w k$ & 4 & $0,1,2,3 w k$ & 0 & Y \\
\hline $150 \mathrm{~g}$ & $\mathrm{Cn}$ & Rt & NiTi pushsp & $\mathrm{N}$ & $12 \mathrm{wk}$ & 5 & $0,1,4,8,12 w k$ & 0 & Y \\
\hline $100 \mathrm{~g}$ & $\mathrm{Cn}$ & Rt & NiTi pushsp & $N$ & $12 \mathrm{wk}$ & 5 & $0,1,4,8,12 \mathrm{wk}$ & 0 & $\mathrm{~N}$ \\
\hline $50-75 g$ & $\mathrm{Cn}$ & LV & NiTi wi & Y & $28 d$ & 5 & $0,7,14,21,28 d$ & 0 & N \\
\hline NM & $\mathrm{Cn}$ & LV & NiTi wi & NM & $6 \mathrm{~m}$ & 5 & $0,1 \mathrm{wk}, 1 \mathrm{~m}, 3,6 \mathrm{~m}$ & 0 & Y \\
\hline $250 \mathrm{~g}$ & $\mathrm{Cn}$ & Rt & $\begin{array}{l}\text { NiTi open } \\
\text { coil sp }\end{array}$ & NM & $28 d$ & 3 & $0,14,28 d$ & 0 & $\mathrm{~N}$ \\
\hline $150 \mathrm{~g}$ & $\operatorname{ImF}$ & Rt & E ch & NM & $28 d$ & 6 & $0,1,7,14,21,28 d$ & 0 & Y \\
\hline $16 \mathrm{~N} /$ turn & $\operatorname{ImF}$ & $M \times \operatorname{Exp}$ & Hyr & $2 / d$ & $6 m$ & 3 & $0,3,6 \mathrm{~m}$ & ० & N \\
\hline $250 \mathrm{~g}$ & $\mathrm{Cn}$ & Rt & NiTi sp & NM & 4 wk & 6 & $0,1 h, 7,14,21,28 d$ & 0 & $N$ \\
\hline NM & $\mathrm{Cn}$ & Rt & NiTi sp & NM & $4 w k$ & 6 & $0,1 h, 7,14,21,28 d$ & 0 & $\mathrm{~N}$ \\
\hline $125 \mathrm{~g}$ & $\mathrm{Cn}$ & Rt & NiTi sp & NM & $21 d$ & 6 & $0,1 \mathrm{~h}, 1,7,14,21 \mathrm{~d}$ & 0 & $\mathrm{~N}$ \\
\hline $250 \mathrm{~g}$ & $\mathrm{Cn}$ & Rt & NiTi sp & $N$ & 4 wk & 6 & $0,1 \mathrm{~h}, 1,2,3,4 \mathrm{wk}$ & 0 & $\mathrm{~N}$ \\
\hline $200 \mathrm{cN}$ & Imf vs Cn & Rt & Hycon, TieB & Screw 2/wk & $28 d$ & 6 & $0,1 \mathrm{hr}, 7,14,21,28 \mathrm{~d}$ & 0 & Y \\
\hline NM & $\mathrm{Cn}$ & Aln & NiTi wi & NM & $\begin{array}{c}\text { Completion } \\
\text { of Aln }\end{array}$ & 4 & $0,1 \mathrm{~h}, 1 \mathrm{wk}$, completion of Aln & 0 & $N$ \\
\hline $400 \mathrm{~g}$ & Imf & Mx Exp & Hyr & $1 / 3 m$ & $1 y$ & 4 & $0,2 w k, 4 w k, 1 y$ & 0 & $\mathrm{~N}$ \\
\hline NM & $\mathrm{Cn}$ & Aln & $\begin{array}{l}\text { MSSS, HANT, } \\
\text { SE wi }\end{array}$ & N & $14 d$ & 4 & $0,2 h, 7,14 d$ & 0 & Y \\
\hline NM & $\mathrm{Cn}$ & Aln & $\begin{array}{c}\text { MSNiTi, HANT, } \\
\text { SE wi }\end{array}$ & $N$ & $14 d$ & 4 & $0,2 h, 7,14 d$ & 0 & Y \\
\hline NM & $\mathrm{Cn}$ & Aln & NiTi wi & $\mathrm{N}$ & 4 wk & 6 & $0,1 \mathrm{~h}, 1,2,3,4 \mathrm{wk}$ & 0 & $\mathrm{~N}$ \\
\hline
\end{tabular}

CRP- C Reactive Protein, hm-humidity, sc-specification, ins-insertion, MB-Mesio-buccal, ML-Mesio-lingual, DP-Disto-palatal, DB- Disto-buccal, df-differentiation gp-group, cmp-compression, kPa-kilopascal, mx-maximum, gw-growth, Oc-osteoclast, Ix- Index, Bu Tp-buccal tipping, C-canine, Clf-cleft, I-incisor, NA-not applicable, wk-week, crw-crowding, minm-minimum, bimax-bimaxillary, wi-wire, lig-ligature, Ad-adult, RANKL-receptor antagonist nuclear kappa ligand, OPG-osteoprotegerin, IL-_1RA-interleukin 1 receptor antagonist, therm-thermoplastic, t-PA-plasminogen, TNF $\alpha$-tumour necrosis factor, TIMP-Tissue inhibitor metalloproteinase, MCP- Methyl-accepting chemotaxis protein, MPO-myeloperoxidase, ortho-orthodontic, cys-cysteine, cN-centinewton, TSP-thrombospondin 1, NGAL-neutrophil gelatinase-associated lipocalin, ACP-acyl carrier protein, CS-chondroitin sulphate, GM-CSF- Granulocyte-macrophage colony-stimulating factor, IFN $\gamma$-Interferon gamma, MIP-Macrophage inflammatory protein, $\beta$ G-beta globulin, PAI-plasminogen activator inhibitor, EGF-Epidermal growth factor, dst-distalisation, Intr-intrusion, aln-alignment, cst-constriction, AL-after loading, BL-before loading, Mx constr- Maxillary constriction, Exp- Expansion, Hyr- Hyrax, LB-laceback, TB-Tie back, SE-superelastic NiTi, HANT- heat-activated NiTi, MSSS- multistranded stainless steel. 
Table 4 - Oral hygiene regimen.

\begin{tabular}{|c|c|c|c|c|c|c|c|}
\hline Ref No. & Oral px (Pre t/t) & $\begin{array}{c}\text { Oral px } \\
\text { (Every ob po) }\end{array}$ & $\begin{array}{l}\text { Oral hy instr/ } \\
\text { motiv }\end{array}$ & $M w$ & $\mathrm{fq} / \circ \mathrm{mw} / \mathrm{d}$ & $\begin{array}{l}\text { asm for gv } \& \\
\text { pd in (pre } t / t)\end{array}$ & At every ob po \\
\hline 7 & Y & NM & Y & Cxglu & 2 & Y & Y \\
\hline 8 & Y & NM & NM & NM & NM & Y & NM \\
\hline 9 & NM & NM & Y & 0.15\% Benz HCL/d & $1 / d$ & NM & NM \\
\hline 10 & Y & NM & Y & $0.5 \mathrm{oz}$ of $0.2 \% \mathrm{cx}$ glu & $2 / d$ & NM & NM \\
\hline 11 & Y & NM & NM & NM & NM & Y & NM \\
\hline 12 & NM & NM & NM & NM & NM & NM & NM \\
\hline 13 & Y & Y & Y & NM & NM & NM & NM \\
\hline 14 & Y & NM & Y & NM & NM & Y & Y \\
\hline 15 & Y & NM & NM & NM & NM & Y & NM \\
\hline 16 & Y & Y & Y & NM & NM & Y & Y \\
\hline 17 & Y & Y & Y & 0.5 oz of $0.2 \%$ cx glu & $2 / d$ & Y & Y \\
\hline 18 & NM & NM & NM & NM & NM & Y & Y \\
\hline 19 & Y & Y & Y & $0.5 \mathrm{oz}$ of $0.2 \%$ cx glu & Y & Y & Y \\
\hline 20 & Y & Y & Y & $0.12 \%$ cx glu & $2 / d$ for $4 w k$ & NM & NM \\
\hline 21 & NM & NM & Y & NM & NM & Y & NM \\
\hline 22 & NM & NM & Y & NM & NM & NM & NM \\
\hline 23 & Y & Y & NM & NM & NM & Y & Y \\
\hline 24 & $Y$ & Y & Y & cx glu & $2 / d$ & Y & NM \\
\hline 25 & NM & NM & NM & Benz $\mathrm{HCL}$ & NM & NM & NM \\
\hline 26 & Y & NM & Y & NM & NM & Y & Y \\
\hline 27 & NM & NM & NM & NM & NM & Y & NM \\
\hline 28 & Y & NM & Y & NM & NM & Y & Y \\
\hline 29 & NM & NM & NM & NM & NM & Y & NM \\
\hline 30 & NM & NM & Y & NM & NM & Y & Y \\
\hline 31 & Y & NM & Y & cxglu & NM & Y & Y \\
\hline 32 & NM & NM & NM & NM & NM & Y & Y \\
\hline 33 & $Y$ & NM & Y & NM & NM & Y & Y \\
\hline 34 & NM & NM & Y & NM & NM & NM & NM \\
\hline 35 & Y & NM & Y & NM & NM & NM & NM \\
\hline 36 & Y & NM & NM & NM & NM & NM & NM \\
\hline 37 & Y & Y & Y & $N$ & $N$ & Y & Y \\
\hline 38 & Y & Y & Y & $N$ & $N$ & Y & Y \\
\hline 39 & Y & Y & Y & $\mathrm{N}$ & $N$ & Y & Y \\
\hline 40 & Y & Y & Y & $\mathrm{N}$ & N & Y & $\mathrm{T}$ \\
\hline 41 & Y & Y & Y & $N$ & $N$ & Y & Y \\
\hline 42 & NM & NM & Y & cxglu & $2 / d$ & Y & Y \\
\hline 43 & Y & NM & Y & N(against it) & $N$ & Y & NM \\
\hline 44 & Y & Y & Y & $0.012 \%$ cx glu & $2 / d$ & Y & Y \\
\hline 45 & Y & Y & Y & Cxglu & NM & Y & Y \\
\hline 46 & Y & Y & Y & NM & NM & Y & Y \\
\hline 47 & Y & NM & Y & NM & NM & Y & NM \\
\hline 48 & Y & NM & Y & NM & NM & Y & Y \\
\hline 49 & NM & NM & NM & NM & NM & NM & NM \\
\hline 50 & NM & NM & NM & NM & NM & Y & $Y$ \\
\hline 51 & Y & Y & NM & NM & NM & Y & NM \\
\hline 52 & Y & NM & Y & NM & NM & $Y$ & NM \\
\hline 53 & $Y$ & NM & Y & NM & NM & $Y$ & NM \\
\hline 54 & Y & NM & NM & NM & NM & $Y$ & NM \\
\hline
\end{tabular}

A-article, Mw-mouth wash, fq/o-frequency of, d-day, px-prophyaxis, t/t-treatment, ob-observation, po-point, asm-assessment, gv-gingival, pd-periodontal, ininflammation, cx glu-chlorhexidine gluconate, Y-yes, NM-not mentioned, N-no, h-hour, Benz HCL-benzydamine hydrochloride, wk-week, hy-hygiene, instrinstructions, motiv-motivation. 
Table 5 - GCF characteristics.

\begin{tabular}{|c|c|c|c|c|c|c|c|c|c|c|c|c|}
\hline Ref No. & Time & tp & $\mathrm{hm}$ & $\mathrm{mt} / \circ \mathrm{cl}$ & ins (in mm) & $\mathrm{du} / \circ \mathrm{mm}$ & rep mm & $1 / 0 \mathrm{~mm}$ & $\mathrm{mt} / \circ \mathrm{mm}$ & tp of st & $\mathrm{mt} / \circ \mathrm{al}$ & prec \\
\hline 7 & NM & $30 \%$ & $21^{\circ} \mathrm{C}$ & PP & NM & $30 s$ & NM & NM & PT6000 & NM & ELISA & $\mathrm{pg} / 30-\mathrm{s}$ \\
\hline 8 & NM & NM & NM & PP & NM & $30 \mathrm{~s}$ & 4 & NM & NM & $-70^{\circ} \mathrm{C}$ & $\mathrm{SP}$ & 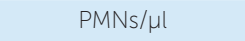 \\
\hline 9 & NM & NM & NM & PP & $1 \mathrm{~mm}$ & $1 \mathrm{~min}$ & 3 & $1 \mathrm{~min}$ & NM & NM & SP & $\mathrm{IU} / 1 \mu \mathrm{l}$ \\
\hline 10 & NM & NM & NM & PP & NM & $1 \mathrm{~min}$ & NM & NM & PT 8000 & $-70^{\circ} \mathrm{C}$ & $\mathrm{SP}$ & $I U / L$ \\
\hline 11 & NM & NM & NM & PP & $1-2 \mathrm{~mm}$ & $1 \mathrm{~min}$ & 3 & NM & NM & $-20^{\circ} \mathrm{C}$ & ELISA & $\begin{array}{l}\text { LDH, AST-mlU/ml, } \\
\text { TRAP, ALP-ng/ml }\end{array}$ \\
\hline 12 & NM & NM & NM & PP & NM & $30 \mathrm{~s}$ & 4 & NM & NM & $-70^{\circ} \mathrm{C}$ & $\mathrm{SP}$ & $\mathrm{U} / 100 \mathrm{ml}$ \\
\hline 13 & NM & NM & NM & PP & NM & $30 s$ & 3 & $1 \mathrm{~min}$ & PT 8000 & $-20^{\circ} \mathrm{C}$ & ELISA & $\mathrm{ng} / \mathrm{ml}$ \\
\hline 14 & NM & NM & NM & $\mu P$ & NM & $5 \mathrm{~min}$ & NM & NM & NM & $-70^{\circ} \mathrm{C}$ & SP & $\mathrm{U} / \mu \mathrm{l}$ \\
\hline 15 & NM & NM & NM & $\mathrm{PP}$ & NM & $1 \mathrm{~min}$ & NM & NM & NM & $-80^{\circ} \mathrm{C}$ & QAK & $\mathrm{pg} / \mathrm{ml}$ \\
\hline 16 & NM & NM & NM & PP & $1 \mathrm{~mm}$ & $30 s$ & NM & NM & NM & $-30^{\circ} \mathrm{C}$ & SP & $\mu \mathrm{g} / \mathrm{ml}$ \\
\hline 17 & NM & NM & NM & $\mathrm{PP}$ & NM & $30 \mathrm{~s}$ & NM & NM & NM & $-70^{\circ} \mathrm{C}$ & IA & $\mathrm{pg} / \mathrm{site}$ \\
\hline 18 & NM & NM & NM & PP & NM & $30 \mathrm{~s}$ & NM & NM & PT 8000 & NM & LMAT & $\mathrm{pg} / \mathrm{ml}$ \\
\hline 19 & NM & NM & NM & PP & NM & $1 \mathrm{~min}$ & 5 & NM & NM & NM & $\mathrm{SP}$ & $\mu \mathrm{molU} / \mathrm{L}$ \\
\hline 20 & NM & NM & NM & PP & $1 \mathrm{~mm}$ & NM & NM & NM & PT 8000 & $-80^{\circ} \mathrm{C}$ & $\mathrm{mb}-\mathrm{IA}$ & $\mathrm{pg} / \mathrm{site}$ \\
\hline 21 & NM & NM & NM & PP & NM & $30 \mathrm{~s}$ & NM & NM & PT8000 & $-80^{\circ} \mathrm{C}$ & Flr & Cp; $\mu \mathrm{U} / \mu \mathrm{l}, \mathrm{Cys} ; \mathrm{ng} / \mu \mathrm{l}$ \\
\hline 22 & 9am & $20^{\circ} \mathrm{C}$ & $40 \%$ & PP & NM & $30 \mathrm{~s}$ & NM & NM & NM & $-70^{\circ} \mathrm{C}$ & WB & NM \\
\hline 23 & NM & NM & NM & PP & $1 \mathrm{~mm}$ & $1 \mathrm{~min}$ & 2 & $1 \mathrm{~min}$ & РT8000 & $-30^{\circ} \mathrm{C}$ & ELISA & $\mu g / \mu l$ \\
\hline 24 & NM & NM & NM & $\mu P$ & NM & NM & NM & NM & NM & $-70^{\circ} \mathrm{C}$ & NM & NM \\
\hline 25 & NM & NM & NM & PP & NM & $1 \mathrm{~min}$ & 2 & $5 s$ & NM & $-80^{\circ} \mathrm{C}$ & ELISA & $\mathrm{pmol} / \mathrm{mg}$ \\
\hline 26 & NM & NM & NM & PP & $1 \mathrm{~mm}$ & $30 s$ & NM & NM & NM & $-80^{\circ} \mathrm{C}$ & SP & $m U$ \\
\hline 27 & NM & NM & NM & PP & NM & $3 \min$ & NM & NM & NM & $-20^{\circ} \mathrm{C}$ & WB & $\mu g / l$ \\
\hline 28 & NM & NM & NM & PP & $1 \mathrm{~mm}$ & $10 \mathrm{~s}$ & NM & NM & NM & NM & $\mathrm{SP}$ & mU/sample \\
\hline 29 & NM & NM & NM & PP & $1 \mathrm{~mm}$ & $1 \mathrm{~min}$ & 1 & $30 \mathrm{~s}$ & NM & $-30^{\circ} \mathrm{C}$ & WB & $\mathrm{pU} / \mu \mathrm{l}$ \\
\hline 30 & NM & NM & NM & PP & $1 \mathrm{~mm}$ & NM & NM & $30 \mathrm{~s}$ & NM & NM & SP & mU/sample \\
\hline 31 & NM & $21^{\circ} \mathrm{C}$ & $30 \%$ & PP & NM & NM & NM & $30 \mathrm{~s}$ & PT6000 & $-70^{\circ} \mathrm{C}$ & ELISA & U/30-s GCF sample \\
\hline 32 & NM & NM & NM & PP & $1 \mathrm{~mm}$ & $1 \mathrm{~min}$ & 1 & $30 \mathrm{~s}$ & PT & $-30^{\circ} \mathrm{C}$ & ELISA & $\mathrm{pg} / \mu \mathrm{g}$ \\
\hline 33 & NM & NM & NM & PP & NM & NM & NM & $30 \mathrm{~s}$ & РT8000 & $-20^{\circ} \mathrm{C}$ & ELISA & $\mathrm{pg} / \mu \mathrm{L}$ \\
\hline 34 & NM & NM & NM & $\mathrm{PP}$ & $1-2 \mathrm{~mm}$ & $1 \mathrm{~min}$ & 2 & $1 \mathrm{~min}$ & NM & NM & $\mathrm{SP}$ & $\mu \mathrm{mol} / \mathrm{min}$ \\
\hline 35 & NM & NM & NM & $\mathrm{PP}$ & $1 \mathrm{~mm}$ & NM & NM & $1 \mathrm{~min}$ & NM & NM & $\mathrm{SP}$ & U/mg \\
\hline 36 & NM & NM & NM & PP & $1 \mathrm{~mm}$ & $1 \mathrm{~min}$ & 3 & $1 \mathrm{~min}$ & NM & NM & $\mathrm{SP}$ & U/mg \\
\hline 37 & NM & NM & NM & end PP & $1 \mathrm{~mm}$ & $30 \mathrm{~s}$ & 3 & $90 \mathrm{~s}$ & NM & $-40^{\circ} \mathrm{C}$ & $\mathrm{SP}$ & $\mu \mathrm{mol} / \mathrm{min}$ \\
\hline 38 & NM & NM & NM & end PP & $1 \mathrm{~mm}$ & $30 \mathrm{~s}$ & 3 & $90 \mathrm{~s}$ & NM & $-40^{\circ} \mathrm{C}$ & $\mathrm{SP}$ & $\mathrm{\mu mol} / \mathrm{min}$ \\
\hline 39 & NM & NM & NM & end PP & $1 \mathrm{~mm}$ & $30 \mathrm{~s}$ & 3 & $90 \mathrm{~s}$ & NM & $-40^{\circ} \mathrm{C}$ & $\mathrm{SP}$ & $\mu \mathrm{mol} / \mathrm{min}$ \\
\hline 40 & NM & NM & NM & end PP & $1 \mathrm{~mm}$ & $30 \mathrm{~s}$ & 3 & $1 \mathrm{~min}$ & NM & $4^{\circ} \mathrm{C}$ & $\mathrm{SP}$ & $\mu \mathrm{mol} / \mathrm{min}$ \\
\hline 41 & NM & NM & NM & $\mu P$ & $2 \mathrm{~mm}$ & NM & NM & NM & NM & $-70^{\circ} \mathrm{C}$ & $\mathrm{SP}$ & $\mathrm{U} / \mathrm{mg}$ \\
\hline 42 & NM & NM & NM & PP & $1 \mathrm{~mm}$ & $30 s$ & NM & NM & NM & $-20^{\circ} \mathrm{C}$ & SP & NM \\
\hline 43 & Y & NM & NM & $\mathrm{FP}$ & NM & $1 \mathrm{~min}$ & NM & $5 s$ & NM & $-80^{\circ} \mathrm{C}$ & PNPP kin & NM \\
\hline 44 & NM & NM & NM & $\mathrm{FP}$ & $1 \mathrm{~mm}$ & $30 \mathrm{~s}$ & NM & NM & NM & $-80^{\circ} \mathrm{C}$ & SP & mU/sample \\
\hline 45 & NM & NM & NM & $\mu P$ & NM & NM & NM & NM & NM & NM & $\mathrm{SP}$ & $\mathrm{U} / \mathrm{L}$ \\
\hline 46 & NM & NM & NM & $\mu P$ & NM & NM & NM & NM & NM & NM & SP & $U / L$ \\
\hline 47 & NM & NM & NM & $\mu P$ & NM & NM & NM & NM & NM & $-80^{\circ} \mathrm{C}$ & $\mathrm{SP}$ & umol units/L) \\
\hline 48 & NM & NM & NM & $\mathrm{pp}$ & $1 \mathrm{~mm}$ & $30 \mathrm{~s}$ & NM & NM & NM & NM & $\mathrm{SP}$ & mU/sample \\
\hline 49 & NM & NM & NM & $\mu P$ & NM & NM & NM & $30 \mathrm{~s}$ & NM & NM & $\mathrm{SP}$ & $I U / L$ \\
\hline 50 & Y & NM & NM & $\mathrm{PP}$ & $1 \mathrm{~mm}$ & $30 \mathrm{~s}$ & NM & NM & РT8000 & $-80^{\circ} \mathrm{C}$ & SP & $\mathrm{pg} / \mathrm{mL}$ \\
\hline 51 & NM & NM & NM & end PP & $1 \mathrm{~mm}$ & $30 \mathrm{~s}$ & NM & NM & NM & $-30^{\circ} \mathrm{C}$ & $\mathrm{SP}$ & mU/sample \\
\hline 52 & NM & NM & NM & PP & NM & $30 \mathrm{~s}$ & 4 & $30 \mathrm{~s}$ & NM & $-70^{\circ} \mathrm{C}$ & SP & units/100 $\mu \mathrm{L}$ \\
\hline 53 & NM & NM & NM & $\mathrm{PP}$ & NM & $30 s$ & 2 & $30 \mathrm{~s}$ & NM & $-70^{\circ} \mathrm{C}$ & $\mathrm{SP}$ & units/100 $\mu \mathrm{L}$. \\
\hline 54 & NM & NM & NM & $\mathrm{pp}$ & $1 \mathrm{~mm}$ & $1 \mathrm{~min}$ & NM & NM & NM & NM & SP & $\mathrm{mU} / \mathrm{s}$ \\
\hline
\end{tabular}

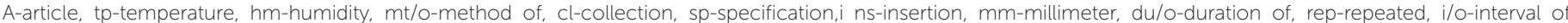

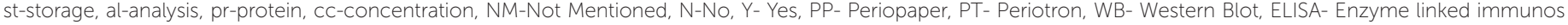

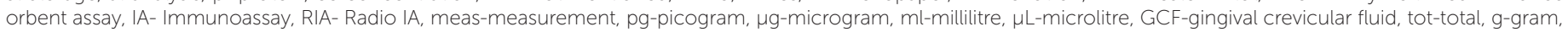

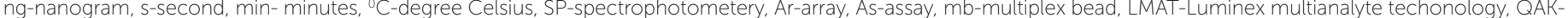

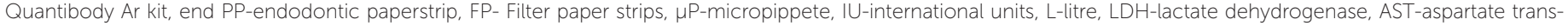

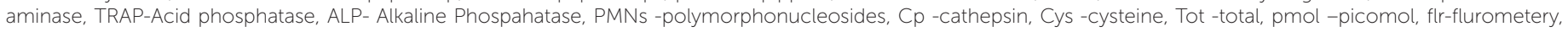
QA- Quantibody assay, PNPP kin- para nitrophenyl phosphate kinetic, pp- paper point. 
Table 6 - Differential expression of enzymes in GCF

\begin{tabular}{|c|c|c|c|c|c|}
\hline Ref No. & sts al ap & cf & Drop outs & Up / down rg & Pk \\
\hline 7 & 1-tailed paired Student t & Y & NM & $\beta G$ : inc & $\begin{array}{l}\text { M-010 } \\
\text { PM-07 } \\
\text { Cl-08 }\end{array}$ \\
\hline 8 & ANOVA, paired $t$ test & Y & $\mathrm{NaM}$ & Inc at $2 \mathrm{~h}$, bas in $7 \mathrm{~d}$ & $2 \mathrm{~h}$ \\
\hline 9 & $\begin{array}{l}\text { (ANOVA) Kolmogorov-Smirnov test, } \\
\text { Paired-samples test }\end{array}$ & Y & N & Inc & 2 wk \\
\hline 10 & $\begin{array}{l}\text { Kolmogorov and Smirnov } \\
\text { (ANOVA) \& Tukey's post-hoc test }\end{array}$ & Y & N & Inc & $14 d$ \\
\hline 11 & $\begin{array}{l}\text { Paired t test } \\
\text { Pearson's cr }\end{array}$ & Y & N & $\begin{array}{c}\text { LDH inc at 2, } 3 \& 4 \mathrm{wk}(100 \mathrm{~g}) \& 1,2 \text { \& } 3 \mathrm{wk}(150 \\
\text { g). AST inc at } 4 \text { \& } 5 \mathrm{wk}(100 \mathrm{~g}) \& 3 \& 4 \mathrm{wk}(150 \\
\text { g). TRAP inc at } 5 \mathrm{wk}(100 \mathrm{~g})\end{array}$ & $\begin{array}{l}\text { AST: } 1 w k \\
\text { TRAP: } 2 w k \\
\text { ALP: } 5 w k\end{array}$ \\
\hline 12 & $\begin{array}{l}\text { Friedman test for intergp \& intragp, Wilcoxon } \\
\text { test for related samples, Kruskal-Wallis test for } \\
\text { independent samples in both gps }\end{array}$ & Y & N & 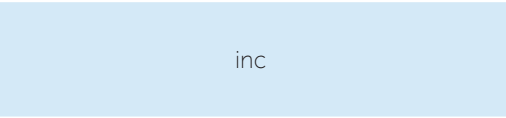 & $2 \mathrm{~h}$ \\
\hline 13 & $\begin{array}{l}\text { Intra gp: Friedman's test, Wilcoxon test } \\
\text { Inter gp: Mann-Whitney U test, Pearson's test }\end{array}$ & Y & N & inc & $\begin{array}{l}\text { MMP9: } 8 \mathrm{~h} \\
\text { MMP9/NGAL: } \\
72 h\end{array}$ \\
\hline 14 & $\begin{array}{l}\text { Intergp: Mann Whitney U test. } \\
\text { Intra gp: Students unpaired t-test }\end{array}$ & Y & N & Inc & $\begin{array}{l}\text { ACP: } 3 d \text { ALP, } \\
\text { AST: } 15 d\end{array}$ \\
\hline 15 & $\begin{array}{c}\text { SAS version } 9.2 \\
\text { proc mixed subroutine }\end{array}$ & Y & N & No sts sn change & NM \\
\hline 16 & $\begin{array}{c}\text { MedCalc software } \\
\text { Intergp: Student's t-test, ANOVA. }\end{array}$ & Y & N & $\begin{array}{l}\text { SL+ NiTi wi: inc } \\
\text { SL+ thrm wi: dec }\end{array}$ & $\begin{array}{l}\text { No sts sn } \\
\text { change }\end{array}$ \\
\hline 17 & Luminex analysis & Y & N & MMP1,3:inc, pk at 24h MMP8: pk at 14d & $24 \mathrm{~h}$ \\
\hline 18 & $\begin{array}{l}\text { Paired non-parametric } \\
\text { Kruskall-Wallis. } \\
\text { Spearman Rank Sum anal }\end{array}$ & Y & NM & Inc & $\begin{array}{c}\text { Exp-TIMP, MMP- } \\
\text { 9:4h } \\
\text { cmp: TIMP-1, } \\
\text { MMP-9:4h, } \\
\text { TIMP-2: } 7 d\end{array}$ \\
\hline 19 & GraphPad®Instat, ANOVA, Friedman & Y & NM & Inc & $14 d, 21 d$ \\
\hline 20 & Friedman, Mann-Whitney & Y & NM & Inc & $1 \mathrm{~h}$ \\
\hline 21 & ANOVA & & NM & $\begin{array}{l}\text { Cp: dec } \\
\text { Cys:inc }\end{array}$ & $1 d$ \\
\hline 22 & ANOVA & Y & NM & Inc & $\begin{array}{l}\text { MMP1-1h } \\
\text { MMP2-1h,8h }\end{array}$ \\
\hline 23 & Mann-Whitney U-tests & N & NM & Inc at $24 \mathrm{~h}$ at $\mathrm{Ixt}>\mathrm{CT}$ & $24 \mathrm{~h}$ \\
\hline 24 & ANOVA \&LSD & Y & NM & Inc & $14 d$ \\
\hline 25 & Friedman test & Y & NM & Dec & ld \\
\hline 26 & $\begin{array}{l}\text { Friedman and Bonferroni-corrected, Wilcoxon } \\
\text { paired signed rank tests }\end{array}$ & Y & NM & Inc & $14 d$ \\
\hline 27 & NM & Y & NM & LVl of MMP-8 inc in $\mid x t>c$ & NM \\
\hline 28 & $\begin{array}{l}\text { Friedman \& Bonferroni-corrected, Wilcoxon paired } \\
\text { signed rank tests }\end{array}$ & Y & NM & Inc & $28 d$ \\
\hline 29 & $\begin{array}{l}\text { Friedman \& Bonferroni-corrected, Wilcoxon paired } \\
\text { signed rank tests }\end{array}$ & Y & NM & Inc & $\begin{array}{c}\operatorname{tn}: 7 d \\
\mathrm{cmp}: 7814 \mathrm{~d}\end{array}$ \\
\hline 30 & Mann Whitney U-test & Y & N & Inc & $24 \mathrm{~h}$ \\
\hline 31 & $\begin{array}{l}\text { Bonferroni-corrected, 1-way repeated measures } \\
\text { ANOVA, paired Student t test }\end{array}$ & Y & NM & Inc & $14 d$ \\
\hline 32 & One-tailed paired Student $t$ test & Y & N & Inc & $\begin{array}{l}\beta G-25 d \\
I L-1 \beta-\end{array}$ \\
\hline 33 & Student's t test & Y & N & IL-1 $\beta$, IL-6, TNF- $\alpha$, EGF, $\beta 2-\mu G$ & inc \\
\hline
\end{tabular}

A -article, sts -statistically, al-analysis, ap -applied, cf -confounders, rg -regulation, Pk-peak, sd-secondary, oc -outcome, cr-correlation, sn -significant, Y-yes, N-no, NM -not mentioned, inc-increase, dec-decrease, fluct -fluctuated, h-hour, mon-month, d-day, wk-week, tot-total, prot - protein, conc-concentration, mg -milligram, ml -millilitre, g-gram, >-greater than, VAS -visual analogue scale, C-canine, mov-movement, b/w-between, cn-continuous, \&-and, F-force, Asc-associated, gen-genetic, GCF-gingival crevicular fluid, compd-compared, B-baseline, IL-interleukin, BG-beta glucoronidase, TNFa-tumour necrosis factor alpha, SD-short duration, LD-long duration, HG-, RDG-, Diff-difference, vol-volume, Rt-retraction, if-inflammation, Avg-average, cyt-cytokine, chemo-chemokine, kwn-known, MOP, Pl-plaque index, BOP-bleeding on probing, Exp-experimental, c-control, Avg-average, Mx-maxilla, ct-contralateral, differen-differentiation, se-separator, gp-group, cmp-compression, tn- tension, kPa-kilopascal, max-maximum, gw-growth, T-tooth, Oc-osteoclast, RDG- Rapid canine distalisation group, HG- hybrid reactor group, Rt- retraction, Aa-Actinobacillus, rd-reading, wi-wire, lig-ligature, Ad-adult, RANKL-receptor an- 
IL-1 $\beta$ sign inc for Mo- O5 to O10 for PM-O6 to 010

For $\mathrm{Cl}-04,06,07,09,010$

$\&$ dec at $\mathrm{O} 2$ for $\mathrm{Mo}, \mathrm{PM}, \mathrm{Cl}$

Inc MPO in saliva at $2 \mathrm{~h}, \mathrm{~B}$ in $7 \mathrm{~d}$

NM

GCF vol inc from $0-21 d$

Sn inc at $14 d$

In saliva:AST inc at 5wk, TRAP at $2 w k, A L P$ at 1 to $5 w k$

Weak $\mathrm{cr}$ b/w enz quantity $\&$ activity

No cr of crw with change in MPO

+ve cr of Ivl in GCF \& saliva

NM

Exp si; Ivl inc on 14d cr with pk in GCF vol

minm \& severe crw: inc from 0 at $2 \mathrm{~h}, 7 \mathrm{~d}, 14 \mathrm{~d}$ in saliva

$\beta G$ inc for Mo- 07 to 010 PM-07, 08, 010

$\mathrm{Cl}-06,07,010$

\& dec at $\mathrm{O} 2$ for Mo, $\mathrm{PM}, \mathrm{Cl}$

LDH inc at 2, 3 \& 4 wk (100 g) \& 1, 2 \& 3wk (150 g)

AST inc at 4 \& $5 \mathrm{wk}(100 \mathrm{~g})$ \& 3 \& $4 \mathrm{wk}(150 \mathrm{~g})$

TRAP inc at $5 \mathrm{wk}(100 \mathrm{~g})$

At $2 h, 7 d$ sn inc from $B$ \& $14 d$

TSP1: inc from B at $8 \mathrm{~h}$ to $72 \mathrm{~h}$, dec at $1 \mathrm{wk}$

NM

Exp si: in Ad, IL-1/1L-1RA dec in 3wk aftr 1st wi lig ado, RANKL/

OPG pk at 6 wk aftr 1 st rect wi lig

Visual pl scr dec sts sn

NM
Strong \& sn cr b/w MMP9/NGAL \& TSP1 in IxT
MMP9: inc from B at 4h, 8h, 1wk, 2wk.

MMP9/NGAL: inc from $B$ at $8 h, 24 h, 72 h$
Inter gp; ACP: pk at 3d ALP, AST: pk at 15d Intra gp ACP, ALP, AST higher in Mx I than Mo b/w Exp \& cT; no sts sn change

B/w Ad \& adol: no sts sn diff

No sts sn change b/w Exp \& cT or within each gp No sts sg diff b/w comp \& tn, MMP1, 3: inc, pk at 24h MMP8: pk at 14d

+ve cr of GCF vol \& PI

at 0 at $\mathrm{tn}, \mathrm{cmp}$

+ ve $c r$ of TNF- $\alpha, I L-1 \beta, I L-8, G M-C S F$ to speed of OTM at $4 \mathrm{~h}$ in

TIMP1 \& 2, MMP-9 inc at $4 \mathrm{~h}$ in Exp

Exp $\mathrm{cr}$ of IL-1 $\beta, \mathrm{IL}-8, \mathrm{TNF}-\alpha$ inc to if at 0 at $\mathrm{cmp}$

NM NM

GCF vol higher in $\mathrm{cmp}$ than th at 21d

GCF vol inc in $1 d$, dec at $1 \mathrm{~m}$

-ve cr in Cp \& Cys Lvl
NM

GCF vol no sn diff at $24 \mathrm{~h}$

NM

Dpd, osteocalcin dec

NM

NM

Aa colonization inc sn on

28d in ExpT \& ct gp

NM

NM

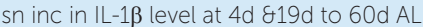

$24 h$
NM

$N M$

NM

NM

NM

NM

NM

NM

NM

NM

NM

NM

NM
Inc at $7,14,21 d$

MMPs inc at $1 \mathrm{~h}$, dec at $24 \mathrm{~h}$

$C p ; d e c$ at $1 d$, inc to $B$ in $1 \mathrm{~m}$

Cys;inc in $1 d$, dec to $B$ in 1 Inc in MMP1 (tn) -1h-3h, (cmp) -1h Inc in MMP2 (tn) - 1h, (comp)-8h Inc at $24 \mathrm{~h}$

Inc in ALP -7, 14 at Ms \& D

Dec from 0 to $28 d$, inc on $7 d$

ct gp greater than Ag gp on 14 d \& 21d

LVI of MMP-8 inc 12 times in $\mid x t>c$

Inc at 28d in Ix T as compd to Ct T \& Ag T

AST: inc in IX T \& ct T as compd to Ag T, inc in IXT as compd to ctT on th si on $14 \mathrm{~d} \&$ on $\mathrm{cmp}$ on $7 \mathrm{~d} \&$

$14 d$, inc in IXT on cmp than tn on $7 d$

$\mathrm{CpB}$ higher at $24 \mathrm{~h}$ at IXT

Sign inc on both $M \& D$ at $1,2,3,4$ wks

inc in $\beta G$ at $25 d$ to $60 \mathrm{~d} \mathrm{AL}$

Intra-grp in Exp: IL-1 $\beta$ inc at $24 \mathrm{~h}>\mathrm{BS}, \mathrm{IL}-6$ inc at $24 \mathrm{~h}>\mathrm{BS}$ or $168 \mathrm{~h}, \mathrm{TNF}-\alpha$ inc at $24 \mathrm{~h}>\mathrm{BS}$ or $168 \mathrm{~h}, \mathrm{EGF}$ inc at $24 \mathrm{~h}>\mathrm{BS}$

Intergrp btw cont $\&$ Exp: IL-1 $\beta$ inc in Exp>cont at 24h, Mean IL-6 in Exp >ant

NM

cont, TNF- in Exp at $24 \mathrm{~h}>$ ant cont. EGF in Exp at $24 \mathrm{hr}>$ ant cont

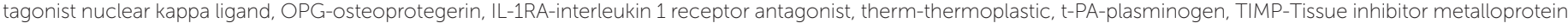

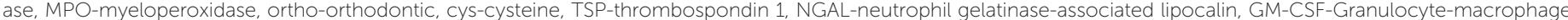

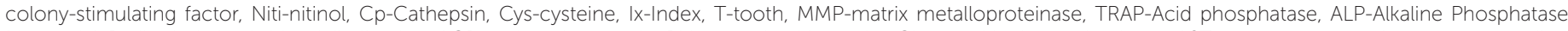

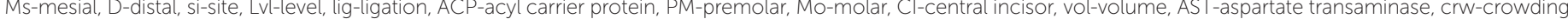

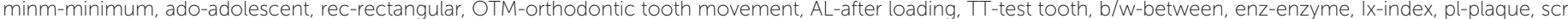

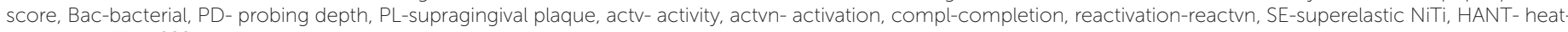
activated NiTi, MSSS- multistranded stainless steel, vol-volume. 
Table 6 - continuation - Differential expression of enzymes in GCF

\begin{tabular}{|c|c|c|c|c|c|}
\hline Ref No. & sts al ap & cf & Drop outs & Up / down rg & Pk \\
\hline 34 & ANOVA & Y & NM & Inc & $7 d$ \\
\hline 35 & Shapiro-Wilk test & Y & NM & inc & $\begin{array}{l}\text { 100g gp-TRAP- } \\
\text { 3wk } \\
\text { 150g gp-ALP \& } \\
\text { TRAP - } 5 w k\end{array}$ \\
\hline 36 & Student's paired t test & Y & NM & Inc & $\begin{array}{c}M: 4 w k \\
D: 1.5 \mathrm{~N}-2 \mathrm{wk}\end{array}$ \\
\hline 37 & Kruskal Walis test. & Y & NM & $\begin{array}{l}\text { TRAP inc in 150g F: Ms site peak 3wk, D site Pk } \\
\text { 4wk den dec } \\
100 \mathrm{~g} \text { F: Ms site 2wk Pk den dec, D site 5wk } \\
\text { 4wk: Lvl in 150g> 100g F (D site) } \\
\text { 5wk: Lvl in 100g>150g F (Dsite) }\end{array}$ & $\begin{array}{l}\text { 150g: D site } \\
-4 w k, \text { Ms site- } \\
\text { 3wk } \\
\text { 100g:D site-5wk. } \\
\text { Ms site-2wk }\end{array}$ \\
\hline 38 & Paired sample t-test & Y & NM & inc, pk at $2 w k$, at $D>M s-1 w k$ & Ms, D Si: 2wk \\
\hline 39 & Paired sample t-test & Y & NM & Dec: 1wk,4wk on Ms, D si & $4 w k$ \\
\hline 40 & Wilcoxon signed rank test & Y & NM & Inc: at 1wk,4wk, stabilised & $4 w k$ \\
\hline 41 & Wilcoxon signed rank test & Y & NM & Inc at $1 w k$, dec in next $3 w k$ & $1 w k$ \\
\hline 42 & unpaired and paired t-test & Y & NM & Inc at archwi >self lig site, inc at 1 wk & $1 w k$ \\
\hline 43 & ANOVA, paired t-test using SPSS & Y & NM & $\begin{array}{c}\text { ALP, ACP inc at } 14 d, 28 d \\
\text { ALP at Ms>D, ACP inc in Ms \&D }\end{array}$ & ALP, ACP : 14d \\
\hline 44 & ANOVA, Student's t-test & Y & NM & $\begin{array}{c}\text { ALP dec, D of } C>\text { Ms of } 2 \text { nd PM on } 1,7,14 \\
\qquad 21,28 d\end{array}$ & Dec \\
\hline 45 & $\begin{array}{l}\text { Friedman test followed by a Bonferroni-corrected } \\
\text { Wilcoxon paired signed rank test }\end{array}$ & Y & Y & ALP inc in $3 m, 6 m$ & Inc \\
\hline 46 & $\begin{array}{l}\text { ANOVA,Tukey's HSD Post-Hoc test, Mann-Whitney } \\
\text { U-test }\end{array}$ & Y & NM & ALP inc $14 d, 28 d$ & $28 d$ \\
\hline 47 & $\begin{array}{l}\text { ANOVA, Independent Samples t-test, Mann- } \\
\text { Whitney U-test }\end{array}$ & Y & NM & $\begin{array}{l}\text { ACP inc both Ms \& D si } \\
\text { D si>M si at 7d, 21d }\end{array}$ & $21 d$ \\
\hline 48 & ANOVA,Tukey HSD & Y & NM & $\mathrm{LDH}$ inc at $T \mathrm{~T}>\mathrm{cT} 7 \mathrm{~d}, 14 \mathrm{~d}, 21 \mathrm{~d}$ & $28 d$ \\
\hline 49 & $\begin{array}{l}\text { Friedman \& Bonferroni-corrected Wilcoxon paired } \\
\text { signed rank tests }\end{array}$ & Y & N & $\begin{array}{l}\text { AST inc from BS in T/t gp from BS to } 2 w k \\
\text { followed by dec } \\
\text { Inc in CC gp from BS to } 1 \text { wk followed by dec } \\
\text { AST level in comp >tn on } 1 w k\end{array}$ & $14 d$ \\
\hline 50 & $\begin{array}{l}\text { One-way ANOVA was used for multiple group and } \\
\text { Student t test for group-wise comparisons }\end{array}$ & Y & N & $\begin{array}{c}\text { inc in ALP b/w } 21 d \text { \& } 28 d \text { : of } 200 \% \text { in active TB } \\
\text { gp, of } 260 \% \text { in Rt screw gp }\end{array}$ & $\begin{array}{c}\text { TB: } 21 d \\
\text { Rt screw: } 28 d\end{array}$ \\
\hline 51 & $\begin{array}{l}\text { independent t tests, _2 tests, or Mann-Whitney, } \\
\text { intraexaminer reliability - concordance } \\
\text { correlation coefficient (CCC) \& Bland-Altman } \\
\text { method }\end{array}$ & N & Y & $\begin{array}{l}\text { MMP8,9, MMP8/TIMP1, MMP9/TIMP1, resistin } \\
\text { at BS>1h>1wK>compl of Aln } \\
\text { CRP, MPO, TIMP, RANKL inc from BS to compl } \\
\text { of Aln } \\
\text { Adiponectin BS<1h<1wk>compl of Aln } \\
\text { Leptin dec from BS to compl of Aln }\end{array}$ & NM \\
\hline 52 & $\begin{array}{l}\text { Fisher's PLSD followed by post hoc, Bonferroni- } \\
\text { Dunn }\end{array}$ & Y & N & $\begin{array}{c}\text { ALP on cmp site: } 0>2 w k>4 w k<1 y \\
\text { tn site: } 0<2 w k<4 w k<1 y\end{array}$ & $\begin{array}{c}\text { tn site: } 1 y \\
\text { cmp site: before } \\
\text { actvn }\end{array}$ \\
\hline 53 & paired $\&$ unpaired ' $t$ ' test and ANOVA. & Y & N & MPO inc from BS to $2 h$ in HANT, SE, MSSS gp & $2 \mathrm{~h}$ \\
\hline 54 & $\begin{array}{c}\text { Chi-square } \\
\text { Student's t-test, and one-way analysis of variance }\end{array}$ & Y & N & $\begin{array}{c}\text { MPO inc from BS to } 2 h \text { in HANT, SE, MSNiTi } \\
\text { gp, HANT>SE \& MS NiTi: } 2 h\end{array}$ & $2 \mathrm{~h}$ \\
\hline 55 & Independent \& paired sample t- test & Y & $\mathrm{N}$ & AST inc from BS to $1 w k$, then dec in Exp gp & $1 w k$ \\
\hline
\end{tabular}

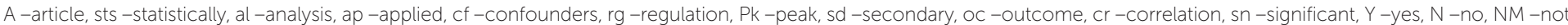

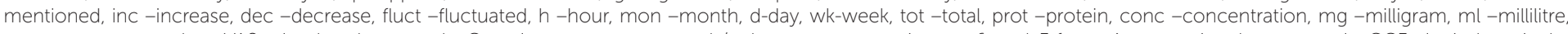

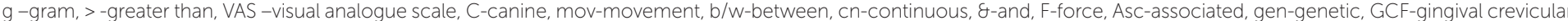

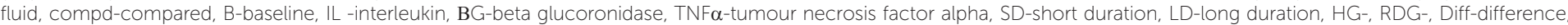

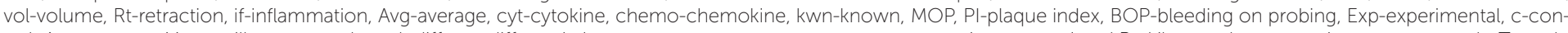

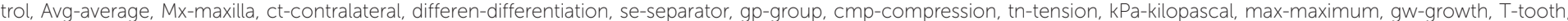

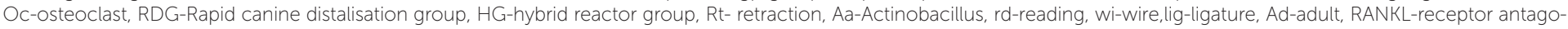


TNF- $\alpha$ in D \& Ms sites of $\pi$ sn higher than both sites of $c$, also $>$. B, inc sn at $1 \mathrm{~h}$ \& 24h. IL-10 dec during Exp period at c \& TT
TRAP5b Level in D \& Ms sites of TT were sn higher than that at both sites of $c T$ compd with $B$ values, inc was sn at $1 \mathrm{~h} \& 24 \mathrm{~h}$.

In $100 \mathrm{~g}$ gp, TRAP sn inc in 3-5 wk compd to TRAPB. ALP \& AST slightly inc. In $150 \mathrm{~g}$ gp, ALP \& TRAP slightly inc compd with their B. AST sn inc in $5 w k$.

$\mathrm{LDH}$ at Ms site in $1.0 \mathrm{~N} \& 1.5 \mathrm{~N}$ gp, inc sn on 4th wk. At D site, LDH with $1.5 \mathrm{~N}$ was higher than $1.0 \mathrm{~N}$ throughout 5 wk of TM. LDH with $1.5 \mathrm{NF}$ inc at both Ms (wk 2) \& D site (wk 3) with sn diff to $1.0 \mathrm{~N} \mathrm{~F}$
Rate of OTM at $150 \mathrm{~g}>100 \mathrm{~g}$
$150 \mathrm{~g}$ gp, Ms si: inc at $3 w k>B S$

At $D$ si: inc at $4 \mathrm{k}>\mathrm{BS}$

TRAP at $150 \mathrm{gm}>100 \mathrm{gm} F$ at $4 \mathrm{wk}$ (D site)

$\begin{array}{cc}\text { NM } & \text { NM } \\ \text { NM } & \text {-ve cr of amt of OTM \& LVl of ALP } \\ \text { NM } & \text { NM } \\ \text { Bac count in archwi>self lig } & \text { +ve cr in self lig \& AST Lvl } \\ \text { NM } & \text { NM } \\ \text { NM } & \text { NM }\end{array}$

Bac count in archwi>self lig

NM

NM

PD with ALP actv

+ve corr of ALP Ivl with time at th si

Inc at $1 w k, 2 w k$ from Bas
Dec at $4 w k$
Inc at $1 w k, 4 w k$, At D si>Ms si
Pk at $1 w k$ at Ix T>cT
Inc at archwi $>$ self lig

NM NM
NM NM NM
NM

GCF flow in $\mathrm{T} / \mathrm{t}=\mathrm{CC}>\mathrm{AC}$ gp

+ve correl of mechanical stress to AST levels, $\mathrm{T} / \mathrm{t}>\mathrm{CC}$
ALP, ACP inc, ALP inc more on $\mathrm{M}$ si

Dec at $D$ of $C>$ Ms of 2nd PM on 1, 7, 14, 21, 28d

Space closure rate, root resorption, Rt, anchorage loss with Hycon screw were assessed

ALP at $3 m, 6 m>c T$

ALP at Ms si of TT>CT at $14 d, 28 d$ At Ms si>Dsi at 14d, 28d

$D$ si>M si at 7d, 21d

TT>cT at 7d, $21 \mathrm{~d}$ at Dsi

LDH inc frm 7d-14d at T,

$\Pi$ T $>$ CT at $7,14,21 d$

sn inc in T/t \&CC vs AC gp: 1, 2, 3, 4w

sn inc in T/t vs CC gp: 1, 2wk

AST level in comp >tn on $1 w k$

Sign diff in ALP on 21d \& 28d b/w TB \& Rt screw gp

resistin at $B S>1 h>1 w k>c o m p l$ of $A l n$

CRP, RANKL inc from BS to compl of Aln

Adiponectin $\mathrm{BS}<1 \mathrm{~h}<1 \mathrm{wk}>\mathrm{compl}$ of Aln

Mediators correl with Aln rate- MPO, RANKL, Leptin, Resistin

MPO at $B S<1 h<1 d<$ compl of Aln

Leptin dec from BS to compl of Aln

NM

NM

NM +ve correl of intermolar distance with ALP level in tn site

MPO in HANT>SE >MSSS th site: 0 (before actvn) $<4 w k, 0<1 y$ cmp site: $0>4 w k, 0<1 y, 2 w k>4 w k$ sn diff in MPO b/w SE \& MSSS : $2 h, 2 w k, b / w$ HANT

\& MSSS:2h, b/w SE \& MSSS:1wk sn diff b/w SE \& MSNiTi: 2h, 1, 2wk, b/w HANT \& MSNiTi:2h

Levels greater in Exp than Cn gp at 1, 2, 3, 4wk

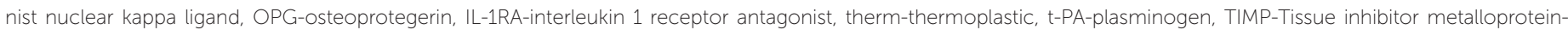

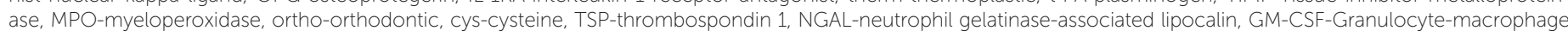

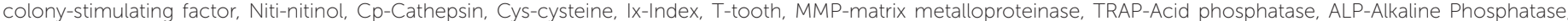

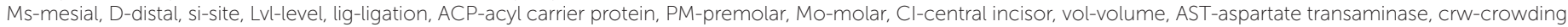

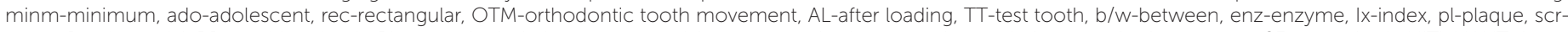

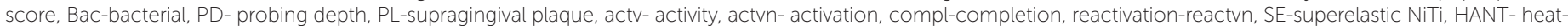
activated NiTi, MSSS- multistranded stainless steel, vol-volume. 
Table 7 -Results of quality assessment of 48 studies for inclusion of studies in the review

\begin{tabular}{|c|c|c|c|c|}
\hline \multirow{2}{*}{ s. No. } & \multirow{2}{*}{ Criteria (29) } & \multicolumn{3}{|c|}{ Response } \\
\hline & & Yes & No & Unclear \\
\hline & I. Study design (18) & & & \\
\hline 1. & Objective: objective clearly formulated & 48 & - & - \\
\hline 2. & Sample size: considered adequate & 2 & - & 46 \\
\hline 3. & $\begin{array}{l}\text { Spectrum of patients representative of } \\
\text { patients receiving the test in practice }\end{array}$ & 48 & - & - \\
\hline 4. & Ethical clearance mentioned & 40 & 8 & - \\
\hline 5. & Selection criteria: clearly described & 48 & - & - \\
\hline 6. & Randomization: stated & 21 & 27 & - \\
\hline 7. & Baseline characteristics: clearly defined & 47 & 1 & - \\
\hline 8. & Control: clearly defined & 46 & - & 2 \\
\hline 9. & $\begin{array}{l}\text { Orthodontic mechanics explained in } \\
\text { sufficient detail to permit replication of } \\
\text { experiment }\end{array}$ & 45 & 1 & 2 \\
\hline 10. & Orthodontic force: clearly specified & 35 & 12 & 1 \\
\hline 11. & $\begin{array}{l}\text { Description of execution of index test: } \\
\text { sufficient to permit replication of test }\end{array}$ & 45 & - & 3 \\
\hline 12 & $\begin{array}{c}\text { Absence of time difference between index } \\
\text { test \& control: mentioned }\end{array}$ & 36 & 12 & - \\
\hline 13. & $\begin{array}{l}\text { Index test executed at specified time and } \\
\text { environmental conditions }\end{array}$ & 4 & 44 & - \\
\hline 14. & $\begin{array}{l}\text { Use of proper indices for assessment of } \\
\text { gingival \& periodontal status (Pre-treatment) }\end{array}$ & 40 & 8 & - \\
\hline 15. & $\begin{array}{l}\text { Use of proper indices for assessment } \\
\text { of gingival \& periodontal status (at each } \\
\text { observation time) }\end{array}$ & 17 & 29 & 2 \\
\hline 16. & Oral hygiene regime-mentioned & 32 & 3 & 13 \\
\hline 17. & Prophylaxis done (Pre-treatment) & 34 & 14 & - \\
\hline 18. & Prophylaxis done(at each observation time) & 11 & 37 & - \\
\hline
\end{tabular}

\section{Study measurements (3)}

\begin{tabular}{|c|c|c|c|c|}
\hline 1. & GCF handling characteristics: explained & 47 & - & 1 \\
\hline 2. & $\begin{array}{l}\text { Measurement method: appropriate to the } \\
\text { objective }\end{array}$ & 48 & - & - \\
\hline \multirow[t]{2}{*}{3.} & Reliability-adequate level of agreement & 48 & - & \\
\hline & \multicolumn{4}{|l|}{ III. Statistical analysis (5) } \\
\hline 1. & $\begin{array}{l}\text { Dropouts: dropouts included in data } \\
\text { analysis }\end{array}$ & 1 & 47 & - \\
\hline 2. & Statistical analysis: appropriate for data & 48 & & - \\
\hline 3. & $\begin{array}{l}\text { Confounders: confounders included in } \\
\text { analysis }\end{array}$ & - & 48 & - \\
\hline 4. & Statistical significance level: P value stated & 48 & - & - \\
\hline 5. & Confidence intervals provided & 48 & - & - \\
\hline \multicolumn{5}{|c|}{ IV. Study results and conclusions (3) } \\
\hline 1. & Index test compared to baseline & 48 & & \\
\hline 2. & Index test compared to control & 48 & & \\
\hline 3. & Conclusions: specific & 40 & & 8 \\
\hline
\end{tabular}

*Index test: Refers to collection of GCF at each observation interval in treatment teeth. 
and $5 \mathrm{~min}$ in one study each. GCF measurements were repeatedly taken in 18 studies with specified number of intervals, interval of repeat measurements were $30 \mathrm{~s}$ $(n=8), 60 s(n=7), 90 s(n=3)$ and $5 s(n=2)$. Storage of samples was done at $-20^{\circ} \mathrm{C}(\mathrm{n}=5),-30^{\circ} \mathrm{C}(\mathrm{N}=4),-40^{\circ} \mathrm{C}$ $(\mathrm{n}=3),-70^{\circ} \mathrm{C}(\mathrm{n}=11)$ and $-80^{\circ} \mathrm{C}(\mathrm{n}=9)$. Retrieval of GCF from Periopaper was done by Periotron (OraFlow, PlainView, New York, NY, USA) in 11 studies, but not mentioned in 38 studies. Enzymes levels were estimated by ELISA ( $n=8)$, spectrophotometry $(n=30)$, immunoassay $(n=2)$, Luminexmultianalyte technology ( $n=1)$, Quantibody Array kit $(n=1)$, western blotting $(n=3)$, fluorometry $(n=1)$ and para-nitrophenol phosphate kinetic $(n=1)$, but omitted in one study. Protein concentration in GCF was measured in variable units in 38 out of 42 studies.

\section{DISCUSSION}

The findings of the current review are presented in Table 6. It depicts various enzymes released in GCF in a time-dependent manner and also establishes correlations (if any) with levels or type of force applied. In this review, we have tried to establish associations of enzyme levels to magnitude or type of force in each phase of OTM, given by Burstone ${ }^{56}$ in his classic model or four phase time/displacement modification model. ${ }^{57,58}$

An initial upregulation in enzymes for bone resorption and matrix degradation like TRAP, ACP or MMPs and an immediate decrease in bone formative ALP corresponded with Burstone's initial phases of OTM. Different MMPs responsible for extracellular matrix (ECM) breakdown are increased at variable times in OTM, ${ }^{13,15,17,18,20,22,27,50}$ as early as 1 hr or till completion of alignment. ${ }^{50}$ MMP-9 increased in $4 \mathrm{hr}$, peaked at $8 \mathrm{hr}$ using stainless steel ligatures for canine retraction in one study, while MMP9/NGAL ratio peaked in $72 \mathrm{hr}$ in another study. ${ }^{13}$

MMPs also varied with different magnitudes of force as MMP-9 peaked in 4hr in a study using $100 \mathrm{~g}$ force for canine retraction, ${ }^{18}$ compared to another study using $150 \mathrm{~g}$ force in which MMP3, 9 and 13 peaked in $24 \mathrm{hr} .{ }^{20}$ The difference in peaks of various MMPs can be explained on the basis of difference in their roles in bone turnover and remodeling with orthodontic forces. $.^{59} \mathrm{MMP}-9$ is responsible for cleavage of denatured collagen, i.e gelatin; ${ }^{60}$ MMP-13 dissolves native fibrillar collagen; MMP-1 is an in- terstitial collagenase hydrolyzing mainly type III collagen, ${ }^{61}$ and MMP-3 is responsible for activation of MMPs 8 and $9 .{ }^{62}$ Hence peaks of MMP8 and MMP9/ NGAL ratio at $14 \mathrm{~d}^{17}$ and $72 \mathrm{hr},{ }^{13}$ respectively, occur subsequent to peak of MMP-3 in $1 \mathrm{hr} / 24 \mathrm{hr} .{ }^{17,20}$ In vitro studies also support rise in MMPs in orthodontic forces, specifically MMP-1,2 mRNA and protein production in human gingival and pdl fibroblasts ${ }^{63,64}$ and MMP-1,2, 9 in gingival tissue of dogs. ${ }^{60}$

On the other hand, no significant change in MMP levels were seen in control teeth where no orthodontic force was applied. ${ }^{17,22}$ This clearly supports MMPs as key mediators of remodeling in OTM.

MMPs are also shown to vary with site (tension and compression) in a time-dependent manner, as supported by in vitro models on pdl fibroblasts. ${ }^{65,66}$ Current review showed an increase in MMP1,2 in 1-3hr on tension site (TS) of maxillary canine after activation of NiTi spring while in compression (CS), MMP1 increased at $1 \mathrm{hr}$ and MMP2 later, at 8hr. ${ }^{22}$ MMP-9 also increased from 4hr to $7 \mathrm{~d}$ on compression site in another study. ${ }^{13}$ This upsurge in levels indicate initial collagen turnover and disintegration of ECM on both tension and compression sites in initial phases of OTM.

Contrary to the MMPs, CS showed a significant increase in GCF levels of MMP inhibitors, TIMP-1 at $4 \mathrm{hr}$ and TIMP-2 after $7 \mathrm{~d}$ during retraction of canines, coinciding with lag phase where tooth movement slows down. ${ }^{18,50}$ At TS, a significant increase in TIMP1 and 2 levels was seen at 4hr, $7 \mathrm{~d}$ and $42 \mathrm{~d}$. This finding is in agreement with the results of a study by Bildt et $\mathrm{a}^{67}$ where a continuous force with NiTi spring of $150 \mathrm{cN}$ was applied for retraction and an increase in MMP1 and TIMP1 was seen on pooled samples from resorption (corresponding to compression) and apposition side (tension) but no trace of TIMP2 was found. The mechanism of action of TIMP-1 stimulates release of MMP1, ${ }^{68}$ an interstitial collagenase, associated with normal tissue remodeling or stretch of pdl fibers, hydrolysing mainly type III collagen. ${ }^{64}$ Also, TIMP-1 increases in smaller amounts on the site of compression, while retraction due to stimulation of bone resorption but in higher amounts on tension, it decreases bone resorption. ${ }^{67} \mathrm{~A}$ study by Garlet et al. ${ }^{69}$ provided evidence of greater expression of TIMP-1 mRNA on TS and MMP-1 mRNA on CS and TS of experimental teeth compared with the control. 
Besides MMPs, histological studies on rats provide evidence of other enzymes for bone resorption predominant in CS in early phases of OTM followed by bone deposition in TS. ${ }^{70,71} \mathrm{In}$ accordance, the current review also shows resorptive enzyme -ACP in initial 3-5d of tooth movement. ${ }^{14}$ Few studies on retraction with continuous forces document an initial rise in ACP both on TS and CS with a peak in $14 \mathrm{~d}^{42}$ and $21 \mathrm{~d} .{ }^{46}$ Initial resorption is followed by a late phase of bone deposition (7-14d) marked by an increase in bone formative ALP levels, ${ }^{37,45}$ seen both in TS and CS of alveolar wall. Increase in ALP occurs by increasing the local concentration of phosphate ions after hydrolysis of phosphomonoester bonds, thus bone mineralisation. Highest serum ALP activity in humans has been correlated with greatest osteoblastic activity during growth spurts. ${ }^{72,73}$ The current review has 17 studies evaluating ALP in association with type, site and magnitude of force. ALP levels increased at TS in continuous retraction forces by NiTi spring as well as in gradually increasing force from $50 \mathrm{cN}$ to $150 \mathrm{cN}$ at $2 \mathrm{wk}$, showing a predisposition towards bone deposition. ${ }^{9}$ A study in rats supported osteoid deposition in the lacunae on TS in 80-120d. ${ }^{74}$ The current review shows peak in ALP levels at $2 \mathrm{wk}$ on continuous force application of $150 \mathrm{cN}, 100 \mathrm{~g}$ or $150 \mathrm{~g}$ force $e^{9,10,14,24,28,45}$, with greater levels on TS compared to CS. This is followed by fall in ALP levels corresponding to hyalinised tissue removal and initiation of post lag phase. ${ }^{9,24}$ Magnitude of force was another determinant of variation in ALP. Decrease in ALP levels seen at $1 \mathrm{hr}, 1 \mathrm{~d}$ after intrusion by TMA spring is believed to be caused by heavy forces leading to a hyalinised zone. ${ }^{25}$ Conversely, distalisation of molars with heavy $\mathrm{cF}$ of $250 \mathrm{~g}^{31}$ showing high ALP levels at both TS and CS and ALP levels greater in $150 \mathrm{~g}$ than $100 \mathrm{~g}$ force, ${ }^{34}$ were attributed to extensive osteoblast recruitment on application of heavy forces. ${ }^{9}$ One study showing decreased ALP levels on both TS and CS of canine retraction with push coil spring was probably due to combination of bodily and tipping movement, which precludes pure compression and tension areas. ${ }^{38}$ ALP also varied with type of force: one study compared levels in Hycon ${ }^{\circledR}$ screw with active tie-backs for retraction. A significant difference was seen at 3 and 4 wk of retraction with levels in Hycon screw group 260\% higher after one half turn twice weekly activation, compared with 200\% increase in active tie-back group. ${ }^{49}$ This may be ascribed to elas- tomeric force decay to $30-40 \%$ of original force in 3 weeks. Another study on maxillary expansion by hyrax followed by retention noticed fall in ALP levels on CS and TS till four weeks of activation, followed by peak at $1 \mathrm{yr}$ on TS, thus indicating bone apposition during retention period. ${ }^{51}$

Contrary to ALP, TRAP or ACP facilitates dissolution of bone minerals by forming a highly acidic extracellular environment and are potent osteoclast biomarkers expressed in areas of compression. ${ }^{74}$ The present review supports rise in TRAP levels at CS more than TS to reach peak at $1 \mathrm{wk},{ }^{33} 2 \mathrm{wk}^{11}$ and 4-5wk. ${ }^{34,36}$ This is supported by histochemical study by Casa et al, ${ }^{75}$ suggestive of appearance of mononuclear TRAP positive cells on application of forces at $2 \mathrm{wk}$ and multinucleated TRAP positive cells at 3 and 4 wk. Even ACP activity was maximum at $3 \mathrm{~d}$, followed by its reversal, explained by natal release of enzymes from surface of osteoclasts. ${ }^{14}$ A secondary outcome of faster rate of OTM with minimal lateral and apical root resorption was noticed with higher levels of TRAP in $150 \mathrm{~g}$, compared with $100 \mathrm{~g}$ force. ${ }^{34,36}$

The consummation of bone resorption occurs by resolution of organic matrix mediated by lysosomal cysteine protease cathepsin B that is increased 1d after application of $100-150 \mathrm{~g}$ or $250 \mathrm{~g}$ retraction force by $\mathrm{E}$ chain, ${ }^{21,30}$ while levels of inhibitor cystatin decreases in 1d. ${ }^{21}$ In association, plasminogen activator (t-PA) and its inhibitor (PAI) responsible for extravascular fibrinolysis, reach peak at $24 \mathrm{hr}$ only to fall later at $7 \mathrm{~d} .{ }^{23}$

AST is another cytoplasmic enzyme released in extracellular environment after cell membrane lysis following necrosis ${ }^{76}$ and has been evaluated in 10 studies in the current SR. Peak levels of AST were seen at $1 \mathrm{wk},{ }^{11,40,41,54} 2 \mathrm{wk},{ }^{14,48}$ and $4 \mathrm{wk} .{ }^{28,39}$ This may be explained on the basis of increase in AST activity for $14 \mathrm{~d}$ due to hyalinization of $\mathrm{pdl}$ in compression zone, decreased later upon resolution of hyalinized area by macrophages. ${ }^{14}$ The formation of hyalinised zone and cellular necrosis may cause higher levels on CS than TS in retraction cases ${ }^{39,48}$ and also in $150 \mathrm{~g}$ force, compared to $100 \mathrm{~g} .{ }^{11,34}$ But, such sporadic evidence could not be definitive for site predilection. Rather this enzyme has been associated more with destruction of gingival tissues in experimental and chronic periodontitis ${ }^{77}$ and subgingival colonization with arch wire ligation ${ }^{41}$ than orthodontic force application. 
The current review has also monitored LDH, an enzyme released from cytoplasm to extracellular space after cell death in gingivitis or periodontitis ${ }^{78}$ as well as in orthodontic treatment. ${ }^{16,26}$ Variation in LDH levels were recorded with type, magnitude and direction of application of force. Continuous force of $125 \mathrm{~g}$ with NiTi spring showed increase in levels at $7 \mathrm{~d}$ to peak at $14 \mathrm{~d},{ }^{26} 21 \mathrm{~d}^{19}$ and $28 \mathrm{~d},{ }^{48}$ but remained higher in CS than TS at $1.5 \mathrm{~N},{ }^{35}$ thus favouring its release after cell death. Timing of increase varied with force level, with an early increase seen at $2 \mathrm{wk}$ in heavy force of $250 \mathrm{~g}$ applied for molar distalisation. ${ }^{26}$ compared with rise in $3 \mathrm{wk}$ in $125 \mathrm{~g}$ force. ${ }^{19,47}$ However no significant difference in LDH levels could be correlated to high friction between selfligating brackets and thermoelastic or superelastic Nitinol wires, as the forces produced by frictional resistance are insufficient for $\mathrm{LDH}$ release. ${ }^{16}$ One study supporting greater LDH levels in teeth undergoing retraction compared with controls was excluded from this review because of its cross-sectional study design. ${ }^{79}$ It supported $\mathrm{LDH}$ as a sensitive marker of the pdl metabolism changes during OTM.

Other inflammatory mediators like MPO and $\beta G$ were also evaluated in this review. MPO released from PMNLs (polymorphonuclear leukocytes) is a sensitive marker for inflammation and pain associated to OTM and showed an early increase at $2 \mathrm{hr} .^{8,12,50,52,53}$ In cases of alignment, the levels of MPO increase from baseline to $1 \mathrm{hr}$ to $1 \mathrm{~d}$ till completion of alignment, correlating it with inflammation caused by NiTi wire alignment. ${ }^{50}$ Studies on MPO also supported superelastic NiTi wires as best alignment wires, giving low continuous force and rapid tooth movement, showing higher MPO levels at $2 \mathrm{hr}$, compared with heat-activated NiTi or multistranded NiTi or stainless steel wires. ${ }^{52,53}$ Studies also mentioned increase in lysosomal enzyme, $\beta G$ released from PMNLs after $14 \mathrm{~d}$ of heavy interrupted force for mid-palatal hyrax expansion in adolescents. ${ }^{7,31}$ However, the levels remained high till $28 \mathrm{~d}$ in retention, probably due to elastic recoil of stretched supracrestal gingival fibers. ${ }^{7,31}$

The risk of bias assessment in QAI though indicated all studies as moderately or highly sensitive, revealed certain strengths and weaknesses of variable study designs (Table 7). While the objectives of the studies, selection criteria and orthodontic mechanics were generally clear, they strikingly lacked sample size calculation with only one study indicating the same. ${ }^{9}$ The authors took 5 as the sample size for inclusion, based on statistician's advice. Randomization of experimental teeth/ side / patients falling into study and control group have been clearly stated in only 21 out of 48 studies, suggesting substantial bias in all studies. The present SR deals with biomarker evaluation in GCF, hence the GCF handling characteristics have been adequate in all studies. However, the specification of time, temperature and humidity at the time of GCF collection was a major shortfall, with only four studies mentioning it. The statistical significance of the results, wherever applicable, have been stated in all the studies, but none of the studies mentioned dropouts or confounders, which might influence the results.

Despite the various shortcomings noticed in the study designs, the current evidence has generated ample evidence related to enzymes in OTM and has also opened new arena for future research in this direction.

Perhaps a most exciting area of research will involve biological basis of tooth movement with different ligation modes of brackets. Further studies could be conducted with LDH as marker for high frictional resistance in different combinations of brackets and wires, as only single study in this SR found no significant change in LDH in initial OTM with self-ligating brackets and superelastic or thermoactive archwire. Another splitmouth study correlating biomarker level with microbial colonization in different ligation modes showed a significantly greater level of AST in arch wire ligation than self-ligation, associated with greater microbial count.

An interesting correlation of MPO with pain was established with an early increase in MPO within $2 \mathrm{hr}$ of force application, coinciding with initial pain incidence in orthodontic patients. $\beta G$ has been explored for its association with the most suitable wires for alignment and could be explored further in different types and magnitudes of forces.

Based on similarity between peri-implant fluid (PIMF) and GCF, the mediators studied in GCF could also be evaluated in PICF to assess stability of contemporary orthodontic anchorage devices, microimplants, as has been suggested by study of interleukin $1 \beta$ in PIMF. ${ }^{80}$

Despite the heterogeneity in study design and categories of enzymes studied in literature, this SR provides an essential overview of the mechanism by which en- 
zymes play a role in bone apposition, resorption as well as ECM degradation. The current SR also correlates mediator levels in GCF with phases of OTM at different magnitudes and types of forces and also ligation modes. It goes a step further in suggesting the potential areas of research in this field, based on individual studies designed for associations of mediator levels with ideal orthodontic force magnitudes, method of ligation and periodontal status, thus setting a direct implication in clinical practice.

\section{CONCLUSIONS}

1. Orthodontic force induces change in levels of multiple enzymes detectable in GCF. These are:

a) cytoplasmic enzymes released in extracellular environment after cell lysis (LDH, AST), b) Inflammatory markers released from PMNs (MPO, $\beta G), c)$ enzymes involved in bone and tissue remodelling by bone resorption (TRAP, ACP), d) bone apposition (ALP) or dissolution of organic matrix (Cp, Cys, tPA, PAI) and e) various categories of MMPs responsible for degradation of ECM (MMP1, 2, 3, 8, 9, 13).

2. Compression sites showed early increase in levels of MMP1, MMP2, TIMP1, MMP9 between 1-4hr, and late peak in TIMP2, TRAP, AST after 7d, 4-5wk and 8-12wk, respectively.

3. Tension sites showed significant increase in ALP after 7d, MMP1 between 1-3hr and TIMP 1 and 2 levels at $4 \mathrm{hr}, 7 \mathrm{~d}$ and $42 \mathrm{~d}$.

4. Distinction between TS and CS could be made with levels of TRAP, AST, LDH, MMP9, being greater on CS than TS, and ALP greater on TS.

5. ALP, TRAP levels were greater in $150 \mathrm{~g}$ force than $100 \mathrm{~g}$ force. An early rise in AST levels was seen in $150 \mathrm{~g}$ force at 3 and $4 \mathrm{wk}$, as compared to $100 \mathrm{~g}$ force at 4 and 5 wk.

6. Mechanical stress with continuous force of $\mathrm{NiTi}$ spring causes increase in MMPs 1, 3 in $24 \mathrm{hr}$ in CS and of ALP as early as $7 \mathrm{~d}$ in TS.

7. No significant association between levels of MMP-9 or AST and growth status could be established as adult and adolescents, gave no significant difference in levels.

\section{Author's contribution (ORCID ${ }^{(D)}$ )}

Priyanka Kapoor (PK): 0000-0002-7676-9743

Nitika Monga (NM): 0000-0001-5206-1006 ${ }^{\circ}$

Om P. Kharbanda (OPK): 0000-0001-8908-4666 ${ }^{(0)}$

Sunil Kapila (SK): 0000-0003-0271-9548 (i)

Ragini Miglani (RM): 0000-0003-2553-0588 (리

Rajeswari Moganty (RM): 0000-0001-9662-5379 (])

Conception or design of the study: PK, NM, OPK. Data acquisition, analysis or interpretation: PK, NM, OPK, SK, RMI, RMO. Writing the article: PK, NM, OPK, SK, RMI, RMO. Critical revision of the article: PK, NM, OPK, SK, RMI, RMO. Final approval of the article: PK, NM, OPK, SK, RMI, RMO. Overall responsibility: PK. 


\section{REFERENCES}

1. Meeran NA. Cellular response within the periodontal ligament on application of orthodontic forces. J Indian Soc Periodontol. 2013:17(1):16-20.

2. Krishnan V, Davidovitch Z. Cellular, molecular, and tissue-level reactions to orthodontic force. Am J Orthod Dentofacial Orthop. 2006 Apr:129(4):469.e1-32

3. Alhadlaq AM. Biomarkers of orthodontic tooth movement in gingival crevicular fluid: a systematic review. J Contemp Dent Pract. 2015;16(7):578-87.

4. Apuzzo F, Cappabianca S, Ciavarella D, Monsurrò A, Silvestrini-Biavati A, Perillo L. Biomarkers of periodontal tissue remodeling during orthodontic tooth movement in mice and men: overview and clinical relevance. Sci World J. 2013 Apr 23:2013:105873.

5. Lilja E, Lindskog S, Hammarström L. Histochemistry of enzymes associated with tissue degradation incident to orthodontic tooth movement. Am J Orthod. 1983:83(1):62-75

6. Kapoor P, Kharbanda OP, Monga N, Miglani R, Kapila S. Effect of orthodontic forces on cytokine and receptor levels in gingival crevicular fluid: a systematic review. Prog Orthod. 2014 Dec 9:15:65

7. Tzannetou S, Efstratiadis S, Nicolay O, Grbic J andLamster I. Comparison of levels of inflammatory mediators IL-1beta and betaG in gingival crevicular fluid from molars, premolars, and incisors during rapid palatal expansion. Am J Orthod Dentofacial Orthop. 2008;133(5):699-707.

8. Marcaccini AM, Amato PA, Leão FV, Gerlach RF, Ferreira JT. Myeloperoxidase activity is increased in gingival crevicular fluid and whole saliva after fixed orthodontic appliance activation. Am J Orthod Dentofacial Orthop. 2010 Nov:138(5):613-6

9. Alswafeeri H, ElKenany W, Mowafy M, Helmy M. Crevicular alkaline phosphatase activity during the application of two patterns of orthodontic forces. J Orthod. 2015;42(1):5-13

10. Alfageeh SA, Anil S. Gingival crevicular fluid flow rate and alkaline phosphatase level as potential marker of active tooth movement. Oral Health Dent Manag. 2014 June:13(2):458-63.

11. Abdul Wahab RM, Abu Kasim N, Senafi S, Jemain AA, Zainol Abidin IZ Shahidan MA, et al. Enzyme activity profiles and ELISA analysis of biomarkers from human saliva and gingival crevicular fluid during orthodontic tooth movement using self-ligating brackets. Oral Health Dent Manag. 2014 June:13(2):194-9

12. Navarro-Palacios A, Garcia-López E, Meza-Rios A, Armendariz-Borunda J Sandoval-Rodríguez A. Myeloperoxidase enzymatic activity is increased in patients with different levels of dental crowding after initial orthodontic activation. Am J Orthod Dentofacial Orthop. 2014 July;146(1):92-7.

13. Surlin P, Silosi I, Rauten AM, Cojocaru M, Foia L. Involvement of TSP1 and MMP9/NGAL in angiogenesis during orthodontic periodontal remodeling Sci World J. 2014;2014:421029

14. Kulal R, Thomas B, Ravi MS, Shetty S. Enzymatic evaluation of gingival crevicular fluid in cleft palate patients during orthodontic treatment: a clinicobiochemical study. J Indian Soc Periodontol. 2013:17(3):319-23.

15. Rody WJ Jr, Wijegunasinghe M, Wiltshire WA, Dufault B. Differences in the gingival crevicular fluid composition between adults and adolescents undergoing orthodontic treatment. Angle Orthod. 2014;84(1):120-6.

16. Bolamperti L, Montanari P, Levrini L, Macchi A, Tagliabue A, Caprioglio A. Tissue response during self-ligating treatment. Prog Orthod 2012:13(2):109-16.

17. Canavarro C, Teles RP, Capelli Júnior J. Matrix metalloproteinases -1, -2, -3, -7, $-8,-12$, and -13 in gingival crevicular fluid during orthodontic tooth movement: a longitudinal randomized split-mouth study. Eur J Orthod .2013;35(5):652-8

18. Grant M, Wilson J, Rock P, Chapple I. Induction of cytokines, MMP9, TIMPS, RANKL and OPG during orthodontic tooth movement. Eur J Orthod. 2013:35(5):644-51

19. Alfageeh SA, Anil S. Lactate dehydrogenase activity in gingival crevicular fluid as a marker in orthodontic tooth movement. Open Dent J. 2011:5:105-9

20. Capelli J Jr, Kantarci A, Haffajee A, Teles RP, Fidel R Jr, Figueredo CM. Matrix metalloproteinases and chemokines in the gingival crevicular fluid during orthodontic tooth movement. Eur J Orthod. 2011;33(6):705-11.

21. Rhee SH, Kang J, Nahm DS. Cystatins and cathepsin B during orthodontic tooth movement. Am J Orthod Dentofacial Orthop. 2009;135(1):99-105.
22. Cantarella G, Cantarella R, Caltabiano M, Risuglia N, Bernardini R Leonardi R. Levels of matrix metalloproteinases 1 and 2 in human gingival crevicular fluid during initial tooth movement. Am J Orthod Dentofacial Orthop. 2006;130(5):568.e11-6.

23. Hoshino-Itoh J, Kurokawa A, Yamaguchi M, Kasai K. Levels of t-PA and PAl-2 in gingival crevicular fluid during orthodontic tooth movement in adults. Aust Orthod J. 2005:21(1):31-7.

24. Batra P, Kharbanda OP, Duggal R, Singh N, Parkash H. Alkaline phosphatase activity in gingival crevicular fluid during canine retraction. Orthod Craniofac Res. 2006:9(1):44-51.

25. Isik F, Sayinsu K, Arun T, Unlüçerçi Y. Bone marker levels in gingival crevicular fluid during orthodontic intrusive tooth movement: a preliminary study. J Contemp Dent Pract. 2015;6(2):27-35

26. Perinetti G, Serra E, Paolantonio M, Bruè C, Meo SD, Filippi MR, et al. Lactate dehydrogenase activity in human gingival crevicular fluid during orthodontic treatment: a controlled, short-term longitudinal study. J Periodontol. 2005:76(3):411-7.

27. Ingman T, Apajalahti S, Mäntylä P, Savolainen P, Sorsa T. Matrix metalloproteinase-1 and -8 in gingival crevicular fluid during orthodontic tooth movement: a pilot study during 1 month of follow-up after fixed appliance activation. Eur J Orthod. 2005:27(2):202-7

28. Perinetti G, Paolantonio M, Serra E, D'Archivio D, D'Ercole S, Festa F et al. Longitudinal monitoring of subgingival colonization by Actinobacillus actinomycetemcomitans, and crevicular alkaline phosphatase and aspartate aminotransferase activities around orthodontically treated teeth. J Clin Periodontol 2004:31(1):60-7

29. Sugiyama Y, Yamaguchi M, Kanekawa M, Yoshii M, Nozoe T, Nogimura A, Kasai $K$. The level of cathepsin B in gingival crevicular fluid during human orthodontic tooth movement. Eur J Orthod. 2003:25(1):71-6.

30. Perinetti G, Paolantonio M, D'Attilio M, D'Archivio D, Tripodi D Femminella $B$, et al. Alkaline phosphatase activity in gingival crevicular fluid during human orthodontic tooth movement. Am J Orthod Dentofacial Orthop. 2002;122(5):548-56

31. Tzannetou S, Efstratiadis S, Nicolay O, Grbic J, Lamster I. Interleukin-1 beta and beta-glucuronidase in gingival crevicular fluid from molars during rapid palatal expansion. Am J Orthod Dentofacial Orthop. 1999:115(6):686-96

32. Uematsu S, Mogi M, Deguchi T. Interleukin (IL)-1 beta, IL-6, tumor necrosis factor-alpha, epidermal growth factor, and beta 2-microglobulin levels are elevated in gingival crevicular fluid during human orthodontic tooth movement. J Dent Res. 1996:75(1):562-7.

33. Karaduman B, Uraz A, Altan GN, Tuncer BB, Alkan O, Gönen S, et al. Changes of tumor necrosis factor- $\alpha$, interleukin-10, and tartrate-resistant acid phosphatase $5 \mathrm{~b}$ in the crevicular fluid in relation to orthodontic movement. Eur J Inflamm. 2015:13(1):3-13

34. Wahab RMA, Yamamoto R, Sintian A, Kasim NA, Abidin IZZ, Senafi S, et al. The effects of orthodontic forces during canine retraction using self- ligating brackets on gingival crevicular fluid enzyme activity, canine movement and root resorption. Sains Malaysiana. 2015;44(2):249-56

35. Ariffin SHZ, Kasim NA, Wahab RMA, Jemain AA. Lactate dehydrogenase activity during tooth movement under $1.0 \mathrm{~N}$ and $1.5 \mathrm{~N}$ continuous force applications. Sains Malaysiana. 2013:42(1):99-105.

36. Wahab RMA, Dasor MM, Senafi S, Abdullah AAA, Jemain AA, Kasim NA et al. Crevicular tartrate resistant acid phosphatase activity and rate of tooth movement under different continuous force applications. African J Pharmacy Pharmacol. 2011;5(20):2213-9

37. Abdullah AAA, Wahab RMA, Ariffin SHBZ. Pattern of crevicular alkaline phosphatase during orthodontic tooth movement: leveling and alignment stage. Sains Malaysiana. 2011:40(10):1147-51.

38. Asma AAA, Rohaya MAW, Hisham ZAS. Crevicular alkaline phosphatase activity during orthodontic tooth movement: canine retraction stage. J Med Sci. 2008:8(3):228-33.

39. Rohaya MAW, Hisham ZAS, Khazlina K. The activity of aspartate aminotransferase during canine retraction (bodily tooth movement) in orthodontic treatment. J Med Sci. 2008;8:553-8. 
40. Rohaya MAW, Hisham ZAS, Khazlina K. Preliminary study of aspartate aminotransferase activity in gingival crevicular fluids during orthodontic tooth movement. J Applied Sci. 2009;9(7):1393-96.

41. Hassan KS, Alagl SA, Ali I. Periodontal status following self-ligature versus archwire ligation techniques in orthodontically treated patients: Clinical, microbiological and biochemical evaluation. Orthod Waves. 2010;69:164-70

42. Farahani M, Safavi MS, Dianat O, Tusi SK, Younessian F. Acid and alkaline phosphatase levels in gcf during orthodontic tooth movement. J Dent Shiraz Univ Med Sci. 2015;16(3 Suppl): 237-45.

43. Jeyraj Y, Katta AK, Vannala V, Lokanathan D, Reddy SN, Rajasigamani K. Estimation of alkaline phosphatase in the gingival crevicular fluid during orthodontic tooth movement in premolar extraction cases to predict therapeutic progression. J Nat SciBiolMed. 2015;6(2):343-6.

44. Perinetti G, D'Apuzzo F, Contardo L, Primozic J, Rupel K, Perillo L. Gingival crevicular fluid alkaline phosphate activity during the retention phase of maxillary expansion in prepubertal subjects: A split-mouth longitudinal study. Am J Orthod Dentofacial Orthop. 2015 July:148(1):90-6.

45. Arifyezdani A. Alkaline phosphatase activity in gingival crevicular fluid a diagnostic marker to assess human orthodontic tooth movement. Biomed Pharmacol J. 2015:8(Spl. Edn): 293-301.

46. Arifyezdani A. Acid phosphatase activity in gingival crevicular fluid during human orthodontic tooth movement. Biomed Pharmacol J. 2015; 8(Spl. Edn):403-8.

47. Lakshmanan P, Sathya PB, Dilip S, Srinivas S. Evaluation of lactate dehydrogenase levels in gingival crevicular fluid during orthodontic tooth movement an in-vivo study. Res J Pharmaceutical Biol Chem Sci. 2015:6(5):877-88.

48. Perinetti G, Paolantonio M, D'Attilio M, D'Archivio D, Dolci M, Femminella B, et al. Aspartate aminotransferase activity in gingival crevicular fluid during orthodontic treatment. A controlled short-term longitudinal study. J Periodontol. 2003:74(2):145-52.

49. Kalha AS, Kachiwala VA, Govardhan SN, McLaughlin RP, Khurshaid SZ. Redefining orthodontic space closure: sequential repetitive loading of the periodontal ligament-a clinical study. World J Orthod 2010;11(3):221-9.

50. Saloom HF, Papageorgiou SN, Carpenter GH, Cobourne MT. Impact of obesity on orthodontic tooth movement in adolescents: a prospective clinical cohort study. J Dent Res. 2017:96(5):547-54.

51. Delli Mauri A, Petrini M, Vitale D, Tecco S, Festa F, Barbato E, et al. Alkaline phosphatase level in gingival crevicular fluid during treatment with QuadHelix. J Biol Regul Homeost Agents. 2015 Oct-Dec;29(4):1017-23.

52. Fatima A, Talapaneni AK, Saleh A, Sana S, Hussain A. An evaluation and comparison of myeloperoxidase enzymatic activity during initial orthodontic alignment: an in vivo study. J Orthod. 2017 Sept;44(3):169-173

53. Bhasin V, Singh M, Goutam M, Singh S, Nigam AS, Joshi A. Comparative evaluation of myeloperoxidase enzymatic activity in gingival crevicular fluid of subjects having orthodontic treatment by different aligning arch wires. J Contemp Dent Pract. 2017:18(10):977-80.

54. Haq AU, Iqbal J, Hussain K, Munir A, Irfan S. Aspartate aminotransferase activity in gingival crevicular fluid during orthodontic tooth movement. APMC. 2017:11(2):149-53.

55. Lamster IB, Hartley LJ, Vogel RI. Development of a biochemical profile for gingival crevicular fluid. Methodological considerations and evaluation of collagen-degrading and ground substance degrading enzyme activity during experimental gingivitis. J Periodontol. 1985 Nov;56 Suppl 11s:13-21.

56. Burstone CJ. The biomechanics of tooth movement. In: Kraus BS Riedel RA, editors. Vistas in Orthodontics. Philadelphia: Lea \& Febiger; 1962. p. 197-213

57. Pilon JJ, Kuijpers-Jagtman AM, Maltha JC. Magnitude of orthodontic forces and rate of bodily tooth movement: an experimental study in beagle dogs. Am J Orthod Dentofacial Orthop. 1996 Jul;110(1):16-23.

58. van Leeuwen EJ, Maltha JC, Kuijpers-Jagtman AM. Tooth movement with light continuous and discontinuous forces in beagle dogs. Eur J Oral Sci. 1999 Dec;107(6):468-74
59. Apajalahti S, Sorsa T, Railavo S, Ingman T. The in vivo levels of matrix metalloproteinase-1 and -8 in gingival crevicular fluid during initial orthodontic tooth movement. J Dent Res. 2003:82:1018-22.

60. Redlich M, Reichenberg E, Harari D, Zaks B, Shoshan S, Palmon A. The effect of mechanical force on mRNA levels of collagenase, collagen type I, and tissue inhibitors of metalloproteinases in gingivae of dogs. J Dent Res. 2001 Dec;80(12):2080-4.

61. Welgus HG, Jeffrey JJ, Eisen AZ. The collagen substrate specificity of human skin fibroblast collagenase. J Biol Chem. 1981 Sept 25;256(18):9511-5.

62. Beklen A, Tüter G, Sorsa T, Hanemaaijer R, Virtanen I, Tervahartiala T, et al. Gingival tissue and crevicular fluid co-operation in adult periodontitis. J Dent Res. 2006 Jan; 85(1):59-63.

63. Nahm DS, Kim HJ, Mah J, Baek SH. In vitro expression of matrix metalloproteinase-1, tissue inhibitor of metalloproteinase- 1 and transforming growth factor-beta1 in human periodontal ligament fibroblasts. Eur J Orthod. 2004 Apr:26(2):129-35

64. Bolcato-Bellemin AL, Elkaim R, Abehsera A, Fausser JL, Haikel Y, Tenenbaum $\mathrm{H}$. Expression of mRNAs encoding for alpha and beta integrin subunits, MMPs, and TIMPs in stretched human periodontal ligament and gingival fibroblasts. J Dent Res. 2000 Sep;79(9):1712-6.

65. He Y, Macarak EJ, Korostoff JM, Howard PS. Compression and tension: differential effects on matrix accumulation by periodontal ligament fibroblasts in vitro. Connect Tissue Res. 2004;45(1):28-39.

66. Takahashi I, Onodera K, Nishimura M, Mitnai H, Sasano Y, Mitani H. Expression of genes for gelatinases and tissue inhibitors of metalloproteinases in periodontal tissues during orthodontic tooth movement. J Mol Histol. 2006 Nov:37(8-9):333-42

67. Bildt MM, Bloemen M, Kuijpers-Jagtman AM, Von den Hoff JW. Matrix metalloproteinases and tissue inhibitors of metalloproteinases in gingival crevicular fluid during orthodontic tooth movement. Eur J Orthod. 2009 Oct; 31(5):529-35.

68. Clark IM, Powell LK, Cawston TE. Tissue inhibitor of metalloproteinases (TIMP-1) stimulates the secretion of collagenase from human skin fibroblasts. Biochem Biophys Res Commun. 1994 Sept 15:203(2):874-80

69. Garlet TP, Coelho U, Silva JS, Garlet GP. Cytokine expression pattern in compression and tension sides of the periodontal ligament during orthodontic tooth movement in humans. Eur J Oral Sci. 2007 Oct:115(5):355-62

70. Keeling SD, King GJ, McCoy EA, Valdez M. Serum and alveolar bone phosphatase changes reflect remodeling during orthodontic tooth movement. Am J Orthod Dentofacial Orthop. 1993 Apr;103(4):320-6.

71. Whyte MP. Hypophosphatasia and the role of alkaline phosphatase in skeletal mineralization. Endocr Rev. 1994 Aug;15(4):439-61.

72. Savic L, Savic D. Serum calcium and phosphorus concentration and alkaline phosphatase activity in healthy children during growth and development. Med Pregl. 2008 July-Aug;61(7-8):393-9

73. Christenson RH. Biochemical markers of bone metabolism: an overview. Clin Biochem. 1997;30:573-93

74. Rody WJ Jr, King GJ, Gu G. Osteoclasts recruitment to sites of compression in orthodontic tooth movement. Am J Orthod Dentofacial Orthop. 2001 Nov:120(5):477-89.

75. Casa MA, Faltin RM, Faltin K, Arana-Chavez VE. Root resorption on torqued human premolars shown by tartrate resistant acid phosphatase histochemistry and transmission electron microscopy. Angle Orthod. 2006 Nov;76(6):1015-21.

76. Williams DL, Marks V. Biochemistry in clinical practice. London: William Heinemann Medical Books;1983.

77. McCulloch CA. Host enzymes in gingival crevicular fluid as diagnostic indicators of periodontitis (Review). J Clin Periodontol. 1994 Aug:21(7):497-506

78. Yost KG, Mallatt ME, Liebman J. Interproximal gingivitis and plaque reduction by four interdental products. J Clin Dent. 2006:17(3):79-83.

79. Serra E, Perinetti G, D'Attilio M, Cordella C, Paolantonio M, Festa F, et al. Lactate dehydrogenase activity in gingival crevicular fluid during orthodontic treatment. Am J Orthod Dentofacial Orthop. 2003 Aug;124(2):206-11.

80. Monga N, Chaurasia S, Kharbanda OP, Duggal R, Rajeswari MR. A study of interleukin $1 \beta$ levels in peri-mini screw crevicular fluid (PMCF). Prog Orthod. 2014;15(1):30-35 